\title{
Normal approximation for the net flux through a random conductor
}

\author{
James Nolen*
}

June 6, 2104

\begin{abstract}
We consider solutions of an elliptic partial differential equation in $\mathbb{R}^{d}$ with a stationary, random conductivity coefficient. The boundary condition on a square domain of width $L$ is chosen so that the solution has a macroscopic unit gradient. We then consider the average flux through the domain. It is known that in the limit $L \rightarrow \infty$, this quantity converges to a deterministic constant, almost surely. Our main result is about normal approximation for this flux when $L$ is large: we give an estimate of the Kantorovich-Wasserstein distance between the law of this random variable and that of a normal random variable. This extends a previous result of the author [29] to a much larger class of random conductivity coefficients. The analysis relies on elliptic regularity, on bounds for the Green's function, and on a normal approximation method developed by S. Chatterjee [8] based on Stein's method.
\end{abstract}

\section{Introduction}

This paper pertains to solutions of the random partial differential equation

$$
-\nabla \cdot\left(a(x)\left(\nabla \phi(x)+e_{1}\right)\right)+\beta \phi(x)=0, \quad x \in D_{L} \subset \mathbb{R}^{d},
$$

where the coefficient $a(x)=\left(a_{i j}(x)\right) \in\left(L^{\infty}\left(\mathbb{R}^{d}\right)\right)^{d \times d}$ is a stationary random matrix satisfying a uniform ellipticity condition. The parameter $\beta \geq 0$ is deterministic. The set $D_{L}=[0, L)^{d}$ is the domain, and we require that $\phi$ satisfies periodic boundary conditions on the boundary of $D_{L}$. Our main result is about the statistical behavior of the quantity

$$
\Gamma_{L, \beta}=\frac{1}{\left|D_{L}\right|} \int_{D_{L}}\left(\nabla \phi+e_{1}\right) \cdot a(x)\left(\nabla \phi+e_{1}\right)+\beta \phi^{2} d x
$$

for large $L$. Using (1.1) and the periodicity of $\phi$ we see that $\Gamma_{L, \beta}$ may also be written as

$$
\Gamma_{L, \beta}=\frac{1}{\left|D_{L}\right|} \int_{D_{L}} e_{1} \cdot a(x)\left(\nabla \phi(x)+e_{1}\right) d x
$$

This is a random variable, as the coefficient $a(x)$ and the solution $\phi$ are random.

Partial differential equations like (1.1) arise in physical applications where the coefficient $a(x)$ may be modeled best as a random field, due to inherent uncertainty and complexity of the physical medium [36. If we interpret (1.1) in terms of electrical conductivity, then $\phi$ is a potential, $a(x)$ is the conductivity, and the vector field $-a(x)\left(\nabla \phi+e_{1}\right)$ is a current density. The unit vector $e_{1}$ is deterministic, the gradient of the linear potential $x \cdot e_{1}$. Considering (1.3), we interpret $\Gamma_{L, \beta}$ as an

*Department of Mathematics, Duke University, Durham, North Carolina, USA. (nolen@math.duke.edu). 
average flux in the direction $e_{1}$ that results from a macroscopic potential gradient imposed in the direction of $e_{1}$.

The equation (1.1) plays an important role in the homogenization theory for the random elliptic operator $u \mapsto-\nabla \cdot(a(x / \epsilon) \nabla u)$ in the limit $\epsilon \rightarrow 0$ [32, 22]. It is well-known that the homogenized conductivity tensor $\bar{a}$ for that operator can be expressed in terms of functions $\phi$, called "correctors", which solve (1.1) with $e_{1}$ being one of the $d$ standard basis vectors and which have stationary gradient. On the other hand, in a numerical computation of $\bar{a}$ one must approximate the true correctors by solving (1.1) in a bounded domain $D_{L}$ with suitable boundary condition. The parameter $\beta \geq 0$ is a kind of regularizing parameter that sometimes is used in approximation theory. The periodic boundary condition that we impose here is one choice that allows accurate approximation of the effective coefficient $\bar{a}$ in the limit $L \rightarrow \infty[7,31,15]$.

The results of [7, 31] imply that for $\beta \geq 0$ fixed, $\Gamma_{L, \beta}$ converges almost surely, as $L \rightarrow \infty$, to a deterministic constant $\bar{\Gamma}_{\beta}>0$. For $\beta=0$, the limit $\bar{\Gamma}_{0}$ is one of the diagonal entries of the homogenized tensor $\bar{a}$ described above. For finite $L$, it is interesting to understand how $\Gamma_{L, \beta}$ and $\phi$ fluctuate around their means. Our main result is an estimate showing that for $L \gg 1$, the distribution of $\Gamma_{L, \beta}$ is very close to that of a normal random variable. In [29], we proved a similar result under strong assumptions about the random coefficient $a(x)$. In the present paper, however, we develop a more general approach which yields normal approximation for $\Gamma_{L, \beta}$ under much weaker assumptions about the law of $a(x)$.

Before we present the main result and explain its relation to other works, let us define the problem precisely and establish notation.

\section{The random coefficient $a(x)$}

For $L \in \mathbb{Z}^{+}$, let $D_{L}=[0, L)^{d} \subset \mathbb{R}^{d}$ and let $L_{\text {per }}^{\infty}\left(D_{L}\right)$ denote the set of functions in $L^{\infty}\left(\mathbb{R}^{d}\right)$ which are periodic with period $L$ in each direction. That is, for all $f \in L_{p e r}^{\infty}\left(D_{L}\right), f(x+L k)=f(x)$ holds for all $k \in \mathbb{Z}^{d}$ and almost every $x \in \mathbb{R}^{d}$. The coefficient $a(x)$ in (1.1) will be a random symmetric matrix with entries $a_{i j} \in L_{p e r}^{\infty}\left(D_{L}\right)$. Since we will be working with functions that are periodic over $D_{L}$, we use $\operatorname{dist}(x, y)$ to refer to the periodized distance function:

$$
\operatorname{dist}(x, y)=\min _{k \in \mathbb{Z}^{d}} d_{2}(x, y+k L), \quad x, y \in \mathbb{R}^{d},
$$

where $d_{2}(x, y)$ is the standard Euclidean metric in $\mathbb{R}^{d}$. Also, when working in the torus $D_{L}$, we use the notation $B_{r}(x)$ to refer to the ball of radius $r$ in this metric on the torus:

$$
B_{r}(x)=\left\{y \in D_{L} \mid \operatorname{dist}(x, y)<r\right\} .
$$

We suppose that the random nature of $a(x)$ comes from its dependence on a collection of $L^{d}$ independent random variables $Z=\left\{Z_{k}\right\}_{k \in \mathbb{Z}^{d} \cap D_{L}}$ taking values in a set $\mathcal{Z}$, and defined over a probability space $(\Omega, \mathcal{F}, \mathbb{P})$. Thus, $Z: \Omega \rightarrow \mathcal{Z}^{L^{d}}$. We often will write $a(x)$ for $a(x, Z)$, the dependence on $Z$ being understood. Let $\mathbb{E}[f(Z)]$ denote expectation with respect to the probability measure $\mathbb{P}$ defining the law of $Z$. We will make three additional structural assumptions about the random matrix $a(x)$. First, we require that $a(x)$ is statistically stationary with respect to integer shifts in $x$ : for every $k \in \mathbb{Z}^{d}$ and $a(\cdot+k)$ is equal in law to $a(\cdot)$. Second, we suppose boundedness and uniform ellipticity: there are positive constants $a^{*}, a_{*}>0$ such that for any nonzero $\xi \in \mathbb{R}^{d}$

$$
a_{*}|\xi|^{2} \leq \xi \cdot a(x) \xi \leq a^{*}|\xi|^{2}, \quad x \in D_{L}
$$

holds $\mathbb{P}$-almost surely. Third, we suppose that there is a constant $\tau>\sqrt{d}>0$ such that for all $k \in \mathbb{Z}^{d}$

$$
a(x, Z)-a\left(x, Z^{\prime}\right)=0 \quad \text { if } \operatorname{dist}(x, k) \geq \tau
$$


holds whenever $Z_{j}=Z_{j}^{\prime}$ for all $j \neq k$. One consequence of this last assumption is that $x \mapsto a(x, Z)$ does not depend of $Z_{k}$ if $\operatorname{dist}(x, k) \geq \tau$. Moreover, $a(x, Z)$ and $a(y, Z)$ are statistically independent if $\operatorname{dist}(x, y) \geq 2 \tau$. In other words, the dependence of $a(x, Z)$ on $Z$ is local: $a(x, Z)$ depends only on $Z_{j}$ for indices $j \in \mathbb{Z}^{d}$ that are sufficiently near $x \in \mathbb{R}^{d}$.

For clarity, let us highlight some simple examples for which these assumptions hold. First, suppose that $a(x)$ is scalar and has the form of a random checkerboard

$$
a(x)=\sum_{k \in \mathbb{Z}^{d} \cap D_{L}} Z_{k} \mathbb{I}_{Q_{k}}(x \bmod L)
$$

where $\left\{Z_{k}\right\}_{k \in \mathbb{Z}^{d} \cap D_{L}}$ is a family of independent and identically distributed real-valued random variables satisfying $a_{*} \leq Z_{k} \leq a^{*}$ almost surely. The set $Q_{k}=k+[0,1)^{d}$ is the unit cube with a corner at $k \in \mathbb{Z}^{d}$, and $(x \bmod L)$ denotes the point $\left(x_{1} \bmod L, \ldots, x_{d} \bmod L\right) \in D_{L}$. So, $a(x)$ is a piecewise constant function, taking random values on the cubes $Q_{k}$. It is also periodic over $D_{L}$. In this example, $\mathcal{Z}=\left[a_{*}, a^{*}\right]$, but we need not make any further assumptions about regularity of the law of $Z_{k}$, as was required in [29].

In the next example, $a(x, \omega)$ is scalar and represents pores of conductivity $a^{*}$ distributed randomly within a material having background conductivity $a_{*}>0$. The pores are spheres having random radii, whose centers are determined by a Poisson point process with intensity $\mu>0$. To construct such a conductivity function, let $\left\{X_{j}^{k} \mid k \in \mathbb{Z}^{d}, j \in \mathbb{N}\right\}$ be a collection of independent random variables that are each uniformly distributed on the cube $Q_{0}=[0,1)^{d}$. Let $\left\{N_{k}\right\}_{k \in \mathbb{Z}^{d}}$ be an independent set of Poisson random variables with mean $\mu>0$, defined on the same probability space. That is, $\mathbb{P}\left(N_{k}=n\right)=(n !)^{-1} e^{-\mu} \mu^{n}$ for $n=0,1,2, \ldots$ The random integer $N_{k}$ will be the number of pores with centers in the cube $Q_{k}=k+[0,1)^{d}$. The random measure

$$
\rho_{k}(A)=\sum_{j=1}^{N_{k}} \mathbb{I}_{A}\left(k+X_{j}^{k}\right)
$$

on Borel sets $A \subset Q_{k}$ is a homogeneous Poisson point process on $Q_{k}$ with intensity $\mu$. Let $\left\{R_{j}^{k} \mid k \in\right.$ $\left.\mathbb{Z}^{d}, j \in \mathbb{N}\right\}$ be an independent collection of identically distributed, real-valued random variables such that $\mathbb{P}\left(0<R_{j}^{k} \leq R_{\max }\right)=1$ for some constant $R_{\max }$; these are the radii of the pores. Finally, we define

$$
a(x)=a_{*}+\left(a^{*}-a_{*}\right) \min \left(1, \sum_{k \in \mathbb{Z}^{d} \cap D_{L}} \sum_{j=1}^{N_{k}} \mathbb{I}_{B_{R_{j}^{k}}\left(X_{j}^{k}\right)}(x-k)\right) .
$$

Thus, $a(x)=a^{*}$ if and only if

$$
\operatorname{dist}\left(x,\left(k+X_{j}^{k}\right)\right)<R_{j}^{k}, \quad \text { for some } j \leq N_{k} \text { and } k \in \mathbb{Z}^{d} \cap D_{L} .
$$

Otherwise, $a(x)=a_{*}$. In this case, the random variables $\left\{Z_{k}\right\}_{k \in \mathbb{Z}^{d} \cap D_{L}}$ are the collections of (shifted) pore centers and radii: $Z_{k}=\left\{\left(X_{j}^{k}, R_{j}^{k}\right) \mid 0 \leq j \leq N_{k}\right\}$, and we may take the set $\mathcal{Z}$ to be the set of all finite sequences $\left(\left(x_{1}, r_{1}\right), \ldots,\left(x_{n}, r_{n}\right)\right)$ where $x_{i} \in Q_{0}$ and $r_{i} \in\left(0, R_{\max }\right)$. Recalling (1.5)), we see that $a \in L_{p e r}^{\infty}\left(D_{L}\right)$ almost surely, and the stationarity property holds. The condition $\mathbb{P}\left(R_{j} \leq R_{\max }\right)=1$ guarantees that (1.7) holds with $\tau=R_{\max }+\sqrt{d}$. There are many variations of this construction which fit into the framework described above, such as random rods having random orientation and length, as in the experiments described in [2]. 


\section{The energy functional}

Let $H_{p e r}^{1}\left(D_{L}\right)$ denote the set of $L$-periodic functions in $H_{l o c}^{1}\left(\mathbb{R}^{d}\right)$. That is, $\phi \in H_{\text {per }}^{1}\left(D_{L}\right)$ if $\phi \in$ $H_{l o c}^{1}\left(\mathbb{R}^{d}\right)$ and $\phi(x+L k)=\phi(x)$ a.e. $\mathbb{R}^{d}$ for every $k \in \mathbb{Z}^{d}$. If $a_{i j}(x) \in\left(L^{\infty}\left(D_{L}\right)\right)^{d \times d}$ and satisfies (1.6), then there exists a weak solution $\phi \in H_{\text {per }}^{1}\left(D_{L}\right)$ to (1.1):

$$
\int_{D_{L}} \nabla v \cdot a(x)\left(\nabla \phi+e_{1}\right)+\beta \phi v d x=0, \quad \forall v \in H_{p e r}^{1}\left(D_{L}\right) .
$$

For $\beta>0$, the solution is unique. For $\beta=0$, the solution is not unique, but any two solutions in $H_{p e r}^{1}\left(D_{L}\right)$ must differ by a constant. So, under the normalization condition

$$
\int_{D_{L}} \phi(x) d x=0
$$

and for fixed $L$, the solution is unique in $H_{p e r}^{1}\left(D_{L}\right)$ for all $\beta \geq 0$. With $a(x)=a(x, Z)$ satisfying the conditions above, this unique solution $\phi(x)=\phi(x, a, L, \beta)$ depends on the parameters $L$ and $\beta$, on $x \in D_{L}$, and on the random variables $Z=\left\{Z_{j}\right\}_{j \in D_{L} \cap \mathbb{Z}^{d}}$ which determine $a$. The uniqueness of the solution and the stationarity of $a$ implies that $\phi(x)$ is statistically stationary with respect to integer shifts: the law of $\phi(x)$ is the same as that of $\phi(x+k)$ for any $k \in \mathbb{Z}^{d}$.

Having defined both $a(x)$ and $\phi(x)$, we now define the random variable $\Gamma_{L, \beta}$ by (1.2), which is equivalent to (1.3). This also is a function of the $L^{d}$ random variables $\left\{Z_{j}\right\}_{j \in D_{L} \cap \mathbb{Z}^{d}}$. We will use $\Phi_{j}$ and $\Phi_{j}^{\prime}$ to refer to the integrals

$$
\Phi_{j}=\left(\int_{Q_{j}}\left|\nabla \phi(x)+e_{1}\right|^{2} d x\right)^{1 / 2}, \quad \hat{\Phi}_{j}=\left(\int_{B_{\tau}(j)}\left|\nabla \phi(x)+e_{1}\right|^{2} d x\right)^{1 / 2}
$$

which appear frequently in the analysis. Recall that $B_{\tau}(j) \supset Q_{j}$, so $\hat{\Phi}_{j} \geq \Phi_{j}$.

\section{Main result}

Our main result is the following theorem. Suppose $W$ and $Y$ are two real-valued random variables and that $\mu_{W}$ and $\mu_{Y}$ denote the laws on $\mathbb{R}$ of $W$ and $Y$, respectively. The Kantorovich-Wasserstein distance between $\mu_{W}$ and $\mu_{Y}$ is

$$
\begin{aligned}
d_{\mathcal{W}}(W, Y) & =\sup \left\{|\mathbb{E} h(W)-\mathbb{E} h(Y)| \mid\|h\|_{\text {Lip }} \leq 1\right\} \\
& =\sup \left\{\left|\int_{\mathbb{R}} h(w) d \mu_{W}(w)-\int_{\mathbb{R}} h(y) d \mu_{Y}(z)\right| \mid\|h\|_{\text {Lip }} \leq 1\right\} .
\end{aligned}
$$

Theorem 1.1 Let $d \geq 2$. Let $m_{L, \beta}=\mathbb{E}\left[\Gamma_{L, \beta}\right]$ and $\sigma_{L, \beta}^{2}=\operatorname{Var}\left(\Gamma_{L, \beta}\right)$. Let $Y$ denote a standard normal random variable, $N(0,1)$. There is a constant $C>0$ (depending only on $d, a_{*}$, and $a^{*}$ ) and a constant $q>2$ such that

$$
d_{\mathcal{W}}\left(\frac{\Gamma_{L, \beta}-m_{L, \beta}}{\sigma_{L, \beta}}, Y\right) \leq C \frac{L^{-2 d}}{\sigma_{L, \beta}^{3}} \mathbb{E}\left[\Phi_{0}^{6}\right]+C \frac{L^{-3 d / 2} \log (L)}{\sigma_{L, \beta}^{2}} \mathbb{E}\left[\Phi_{0}^{8 q}\right]^{\frac{1}{2 q}}
$$

holds for all $L>2$ and $\beta \geq 0$.

In [29], we obtained a similar result under more restrictive structural assumptions about the law of the coefficient $a$. Specifically, the approach in [29] required that the law of $a(x)$ be obtained by a 
sufficiently smooth mapping of normally distributed random variables. Those assumptions excluded cases like (1.9) where the law of $a(x)$ may have no absolutely continuous part (with respect to Lebesgue measure on $\left.\left[a_{*}, a^{*}\right]\right)$; the assumptions on $a(x, Z)$ in the present setting are significantly less restrictive. Regularity of the law of $a(x)$ in [29] made it possible to differentiate $\Gamma_{L, \beta}$ with respect to the $Z_{k}$ and to apply a "second order Poincaré inequality" developed by Chatterjee in [9]. In the present setting, the more general assumptions on the law of $a(x)$ do not allow us to apply the same approach. Consequently, the proof of Theorem 1.1 is based on a more general normal approximation technique from [8], which is suitable for fully discrete distributions.

The variance $\sigma_{L, \beta}^{2}$ and the moments of the random variable $\Phi_{0}$ which appear in (1.13) depend on both $L$ and $\beta$. If the moments of $\Phi$ are bounded by a constant, independent of $L$ and $\beta$, and if the variance is bounded from below by $\sigma_{L, \beta}^{2} \geq C L^{-d}$, then the bound (1.13) becomes

$$
d_{\mathcal{W}}\left(\frac{\Gamma_{L, \beta}-m_{L, \beta}}{\sigma_{L, \beta}}, Y\right) \leq C L^{-d / 2} \log L
$$

For all dimensions $d \geq 1$, if $\beta \geq \beta_{0}>0$ is bounded away from zero independently of $L$, then all moments $\mathbb{E}\left[\Phi_{0}^{q}\right]$ are bounded independently of $L>1$ (for example, see [29]). If $\beta=0$ or if $\beta>0$ is allowed to vanish as $L \rightarrow \infty$, estimating the moments $\mathbb{E}\left[\Phi_{0}^{q}\right]$ is a delicate issue. Elliptic regularity helps a bit. Meyers' estimate [26] implies that $\nabla \phi \in L^{p^{*}}$ for some $p^{*}>2$. If the ratio $\frac{a^{*}}{a_{*}}$ is sufficiently close to 1 , this $p^{*}$ may be arbitrarily large. As a result, a uniform (in $L$ and $\beta \geq 0$ ) bound on $\mathbb{E}\left[\Phi_{0}^{q}\right]$ follows from this regularity estimate if $\frac{a^{*}}{a_{*}} \approx 1$ (see Lemma 4.3 of [29], for example). This observation goes back to the work of Naddaf and Spencer [28]. On the other hand, without the assumption $\frac{a^{*}}{a_{*}} \approx 1$, the regularity only goes so far. To estimate $\mathbb{E}\left[\Phi_{0}^{q}\right]$ in this situation one can use the arguments developed recently by Gloria and Otto in [17]. In that work, the authors derive variance bounds for a discrete functional similar to $\Gamma_{L, \beta}$, involving an infinite network of random resistors on the bonds of the integer lattice $\mathbb{Z}^{d}$. The PDE (1.1) is replaced by a discrete difference equation on all of $\mathbb{Z}^{d}$, without the periodicity assumption. The stationary potential field $\phi(x)$ is defined at points $x \in \mathbb{Z}^{d}$; the gradient and divergence have interpretations as difference operators. A key point in their analysis is the following bound on moments of the discrete corrector $\phi$ :

$$
\mathbb{E}\left[|\phi(0)|^{q}\right] \leq\left\{\begin{array}{c}
C_{q}, \quad \text { if } d \geq 3 \\
C_{q}|\log (\beta)|^{\gamma_{q}}, \quad \text { if } d=2 .
\end{array}\right.
$$

The constants $C_{q}, \gamma_{q}>0$ are independent of $L>1$ and $\beta>0$. The analysis of [17] can be extended to the present setting (spatial continuum, with periodicity on $D_{L}$ ) to estimate moments of both $\int_{Q_{0}} \phi(x) d x$ and $\Phi_{0}$ (see [29] for some discussion of this). The argument shows that moments of $\int_{Q_{0}} \phi(x) d x$ satisfy the same bound as (1.14), which diverges as $\beta \rightarrow 0$ if $d=2$. On the other hand, $\Phi_{0}$ involves the gradient $\nabla \phi$, and it can be shown that for all $d \geq 2$, all moments $\mathbb{E}\left[\Phi_{0}^{q}\right]$ are bounded independently of $L>1$ and $\beta \geq 0$ [19]. In the discrete setting, the uniform control (in $L$ and $\beta$ ) of $\nabla \phi$ for all $d \geq 2$ was observed already by Gloria, Otto, Neukamm [15] (see Proposition 1 therein).

In view of $\sigma_{L, \beta}^{2}$ appearing in (1.13), let us note that in many cases it is expected that the variance of $\Gamma_{L, \beta}$ is bounded below by $\sigma_{L, \beta}^{2} \geq C L^{-d}$. Indeed, in [29] we proved that this is the case for the random checkerboard model (1.8). This bound is closely related to earlier work of Wehr [37] in the discrete setting. In a forthcoming work [30], we will give a more general sufficient condition under which $\sigma_{L, \beta}^{2} \geq C L^{-d}$ holds for the continuum setting; in particular, this lower bound holds for the coefficient (1.9) constructed from Poisson scatter. It is not known what is the most general class of stationary random fields $a(x, \omega)$ for which the lower bound $\operatorname{Var}\left(\Gamma_{L, \beta}\right) \geq C L^{-d}$ holds. It is conceivable that there are random fields $a(x, Z)$ satisfying both (1.6) and (1.7) such that $\operatorname{Var}\left(\Gamma_{L, \beta}\right)=o\left(L^{-d}\right)$ as $L \rightarrow \infty$, due to some short-range correlation in the variables $Z_{k}$; the same phenomenon is possible 
even for simple averages of $L^{d}$ identically distributed random variables when the variables may be dependent on one-another. For example, suppose $d=1$, and consider the simple case of a sequence of $L$ resistors wired together, in series, having conductivity $a_{1}, a_{2}, \ldots, a_{L}$. The effective conductivity of the series is just the harmonic mean $\Gamma_{L}=\left(L^{-1} \sum_{k=1}^{L} \frac{1}{a_{k}}\right)^{-1}$. Suppose that $\left\{Z_{k}\right\}$ is a sequence of independent, Bernoulli- $p$ random variables. Thus, $\mathbb{P}\left(Z_{k}=1\right)=p, \mathbb{P}\left(Z_{k}=0\right)=1-p$. Suppose that $a_{k}=\left(2+Z_{k+1}-Z_{k}\right)^{-1}$. These $a_{k}$ are dependent, but $a_{k}$ and $a_{j}$ are independent if $|k-j|>1$. However, by definition of $a_{k},\left(\Gamma_{L}\right)^{-1}$ is a telescoping sum, and the variance of the effective conductivity satisfies $\operatorname{Var}\left(\Gamma_{L}\right)=O\left(L^{-2}\right)=o\left(L^{-d}\right)$. Moreover, the distribution of $\Gamma_{L}$ (after normalization) in this simple example is not asymptotically Gaussian.

In addition to the works we have mentioned already, the two works most closely related to Theorem 1.1] are those of Biskup, Salvi, and Wolff [4] and Rossignol [33] regarding discrete resistor network models. By making use of the martingale central limit theorem, Biskup, Salvi, and Wolff [4] have proved a central limit theorem for a discrete quantity similar to $\Gamma_{L, \beta}$ when $\phi$ satisfies linear Dirichlet boundary conditions on a square box, in the regime of small ellipticity contrast (i.e. $\left|\frac{a^{*}}{a_{*}}-1\right|$ is sufficiently small). Using different techniques, including generalized Walsh decomposition and concentration bounds, Rossignol [33] has proved a variance bound and a central limit theorem for effective resistance of a resistor network on the discrete torus. We refer to the recent review paper [3] for many other references on the random conductance model. Also in the discrete setting, Mourrat and Otto [27] have studied the correlation structure of the corrector itself. Delmotte and Deuschel [11] and, more recently, Marahrens and Otto [25] derived some annealed estimates of the mixed second derivatives $\nabla_{x} \nabla_{y} G(x, y)$ of the Green function for the discrete random elliptic operator; as we mention just after Lemma 4.7, there is a step in our proof which involves bounding a similar quantity.

Other works related to Theorem[1.1]include those of Naddaf and Spencer 28, Conlon and Naddaf [10], and Boivin [5] in the discrete case and Yurinskii [38] in the continuum setting; they also derive upper bounds on the variance of quantities similar to $\tilde{\Gamma}_{L, \beta}$ and $\Gamma_{L, \beta}$. Komorowski and Ryzhik [23] have proved some related moment bounds on $\phi$ in the discrete case when $d=1$. If $\beta=0$ and the dimension is $d=1$, then equation (1.1) can be integrated, with the solution $\phi$ written in terms of integrals of $1 / a(x)$. In that case it is known that the solution itself may satisfy a central limit theorem after suitable renormalization; see Borgeat and Piatnitski [6] Bal, Garnier, Motsch, Perrier [1] for precise statement of these results. In the multidimensional setting, however, those techniques do not apply.

The basis for our proof of Theorem [1.1 is a normal-approximation technique of Chatterjee [8] (see Theorem 2.2 therein), based on Stein's method of normal approximation. This tool and related notation is explained in Section 2, In Section 3 we give some deterministic PDE estimates (Cacciopoli's inequality and Meyers' estimate) which are used later in the analysis. Section 4 contains the main argument in the proof of Theorem 1.1. Finally, in Section 5 we prove some facts about the periodic Green's function which are used in Section 4.

A few more comments about notation: throughout the article we will use the convention that summation over indices $j \in D_{L}$ means a summation over $j \in \mathbb{Z}^{d} \cap D_{L}$, with $j \in \mathbb{Z}^{d}$ being understood. For convenience we will also use brackets $\langle f\rangle=\mathbb{E}[f]$ to denote expectation. We also use $C$ to denote deterministic constants that may change from line to line, but do not depend on $L$ or $\beta$.

Acknowledgment. I am grateful to Felix Otto for very helpful discussions. This work was partially funded by grant DMS-1007572 from the US National Science Foundation. 


\section{Normal approximation}

In this section we summarize a general approach to normal approximation based on Stein's method, and we establish some notation that will be used throughout the paper. Suppose $W$ is a random variable with $\mathbb{E}[W]=0$ and $\mathbb{E}\left[W^{2}\right]=1$, and we wish to estimate

$$
\mathbb{E} h(W)-\mathbb{E} h(Y)
$$

where $Y \sim N(0,1)$ is a standard normal random variable, $h$ is a Lipschitz continuous function on $\mathbb{R}$ and satisfying $\left\|h^{\prime}\right\|_{\infty} \leq 1$. Stein's method of normal approximation [35] is based on the following:

Lemma 2.1 (See [8], Lemma 4.2) Suppose $h: \mathbb{R} \rightarrow \mathbb{R}$ is absolutely continuous with bounded derivative, and $Y \sim N(0,1)$. There exists a solution to

$$
\psi^{\prime}(x)-x \psi(x)=h(x)-\mathbb{E}[h(Y)], \quad x \in \mathbb{R}
$$

which satisfies $\left\|\psi^{\prime}\right\|_{\infty} \leq \sqrt{\frac{2}{\pi}}\left\|h^{\prime}\right\|_{\infty}$ and $\left\|\psi^{\prime \prime}\right\|_{\infty} \leq 2\left\|h^{\prime}\right\|_{\infty}$.

Therefore, to estimate (2.15) it suffices to estimate

$$
\langle h(W)-h(Y)\rangle=\left\langle\psi^{\prime}(W)-W \psi(W)\right\rangle=\operatorname{Cov}\left(\left\langle\psi^{\prime}(W)\right\rangle W-\psi(W), W\right)
$$

where $\psi$ solves (2.16). In particular, a bound on $\operatorname{Cov}\left(\left\langle\psi^{\prime}(W)\right\rangle W-\psi(W), W\right)$ which is independent of $h$ satisfying $\left\|h^{\prime}\right\|_{\infty} \leq 1$ will imply a bound on $d_{\mathcal{W}}(W, Y)$. Lemma 2.2 below gives us a way of estimating $\operatorname{Cov}\left(\left\langle\psi^{\prime}(W)\right\rangle W-\psi(W), W\right)$ when $W=f\left(Z_{1}, \ldots, Z_{n}\right)$ is a function of a collection of independent random variables. Suppose $Z=\left(Z_{1}, \ldots, Z_{n}\right) \in \mathcal{Z}^{n}$ is a random $n$-tuple in $\mathcal{Z}^{n}$ having components that are independent, $\mathcal{Z}$ being a given set. Suppose $Z^{\prime}=\left(Z_{1}^{\prime}, \ldots, Z_{n}^{\prime}\right) \in \mathcal{Z}^{n}$ is an independent copy of $Z$. Define

$$
Z^{j}=\left(Z_{1}, \ldots, Z_{j-1}, Z_{j}^{\prime}, Z_{j+1}, \ldots, Z_{n}\right) .
$$

Similarly, for a set $A \subset\{1, \ldots, n\}$, the random $n$-tuple $Z^{A}$ is defined by replacing $Z_{\ell}$ by $Z_{\ell}^{\prime}$, for all indices $\ell \in A$. For any function $f: \mathcal{Z}^{n} \rightarrow \mathbb{R}$, define

$$
\Delta_{j} f(Z)=f\left(Z^{j}\right)-f(Z)
$$

This is a function of both $Z$ and $Z^{\prime}$ and we sometimes write $\Delta_{j} f\left(Z, Z^{\prime}\right)$ to emphasize this point. If $j \notin A$, then define

$$
\Delta_{j} f\left(Z^{A}\right)=f\left(Z^{A \cup\{j\}}\right)-f\left(Z^{A}\right) .
$$

Let $[n]=\{1,2, \ldots, n\}$. The following identity is due to Chatterjee $[8]$ :

Lemma 2.2 (See [8] Lemma 2.3) Suppose $g, f: \mathcal{Z}^{n} \rightarrow \mathbb{R}$ and $\left\langle g(Z)^{2}\right\rangle<\infty,\left\langle f(Z)^{2}\right\rangle<\infty$. Then

$$
\operatorname{Cov}(g(Z), f(Z))=\frac{1}{2} \sum_{j=1}^{n} \sum_{\substack{A \subset[n] \\ j \notin A}} K_{n, A}\left\langle\Delta_{j} g(Z) \Delta_{j} f\left(Z^{A}\right)\right\rangle .
$$

where $K_{n, A}=|A| !(n-|A|-1) ! /(n !)$.

By applying Lemma 2.2 with $g=f$, one can derive the well-known Efron-Stein inequality [12, 34]: 
Lemma 2.3 Suppose $f: \mathcal{Z}^{n} \rightarrow \mathbb{R}$ and $\left\langle f(Z)^{2}\right\rangle<\infty$. Then

$$
\operatorname{Var}(f(Z)) \leq \frac{1}{2} \sum_{j=1}^{n}\left\langle\left|\Delta_{j} f(Z)\right|^{2}\right\rangle .
$$

By applying Lemma 2.2 to $\operatorname{Cov}\left(\left\langle\psi^{\prime}(W)\right\rangle W-\psi(W), W\right)$ in (2.17), one obtains the following normal approximation bound, due to Chatterjee [8]:

Theorem 2.4 (See [8] Theorem 2.2) Let $W=f(Z)$ satisfy $\langle W\rangle=\mu$ and $\left\langle W^{2}\right\rangle=\sigma^{2}$. Then

$$
d_{\mathcal{W}}\left(\frac{W-\mu}{\sigma}, Y\right) \leq \frac{1}{2 \sigma^{3}} \sum_{j=1}^{n}\left\langle\left|\Delta_{j} f(Z)\right|^{3}\right\rangle+\frac{2}{\sigma^{2}} \operatorname{Var}\left(\mathbb{E}\left[T\left(Z, Z^{\prime}\right) \mid Z\right]\right)^{1 / 2}
$$

where $Y \sim N(0,1)$ and

$$
T\left(Z, Z^{\prime}\right)=\frac{1}{2} \sum_{j=1}^{n} \sum_{\substack{A \subset[n] \\ j \notin A}} K_{n, A} \Delta_{j} f(Z) \Delta_{j} f\left(Z^{A}\right)
$$

Our goal will be to prove Theorem 1.1 by applying Theorem 2.4 to the random variable $f(Z)=$ $\Gamma_{L, \beta}$. The term $\operatorname{Var}\left(\mathbb{E}\left[T\left(Z, Z^{\prime}\right) \mid Z\right]\right)$ in Theorem 2.4 can be estimated by the Efron-Stein inequality (2.20). To this end, we introduce a third $n$-tuple $Z^{\prime \prime}=\left(Z_{1}^{\prime \prime}, Z_{2}^{\prime \prime}, \ldots, Z_{n}^{\prime \prime}\right)$ which is an independent copy of $Z$, independent of $Z^{\prime}$. Let us define

$$
Z^{k}=\left(Z_{1}, \ldots, Z_{k-1}, Z_{k}^{\prime \prime}, Z_{k+1}, \ldots\right) .
$$

For any function $g\left(Z, Z^{\prime}\right): \mathcal{Z}^{n} \times \mathcal{Z}^{n} \rightarrow \mathbb{R}$ we define

$$
\Delta_{k} g\left(Z, Z^{\prime}\right)=g\left(Z^{k}, Z^{\prime}\right)-g\left(Z, Z^{\prime}\right) .
$$

In particular, $\Delta_{k} g\left(Z, Z^{\prime}\right)=0$ if $g\left(Z, Z^{\prime}\right)$ does not depend on $Z_{k}$. We use the notation $g^{k}$ to denote the action of replacing $Z_{k}$ by $Z_{k}^{\prime \prime}$ in the argument of $g$ :

$$
g\left(Z, Z^{\prime}\right)^{k}=g\left(Z^{k}, Z^{\prime}\right) .
$$

Thus, $\Delta_{k} g\left(Z, Z^{\prime}\right)=\left(g\left(Z, Z^{\prime}\right)\right)^{k}-g\left(Z, Z^{\prime}\right)$. Let us emphasize that $Z^{k}$ will always refer to (2.22) while $Z^{j}$ refers to (2.18). The $n$-tuples $Z^{j}$ and $Z^{k}$ have the same law, but the $n$-tuple denoted by $Z^{j}$ is not equivalent to $Z^{k}$ even when the values of the indices $k$ and $j$ are the same.

Now, Lemma 2.3 implies

$$
\operatorname{Var}\left(\mathbb{E}\left[T\left(Z, Z^{\prime}\right) \mid Z\right]\right) \leq \frac{1}{2} \sum_{k} \mathbb{E}\left[\left|\Delta_{k} h(Z)\right|^{2}\right]
$$

where $h(Z)=\mathbb{E}\left[T\left(Z, Z^{\prime}\right) \mid Z\right]$ and

$$
\Delta_{k} h(Z)=\mathbb{E}\left[T\left(Z^{k}, Z^{\prime}\right) \mid Z^{k}\right]-\mathbb{E}\left[T\left(Z, Z^{\prime}\right) \mid Z\right]=\mathbb{E}_{Z^{\prime}}\left[T\left(Z^{k}, Z^{\prime}\right)-T\left(Z, Z^{\prime}\right) \mid Z, Z^{k}\right] .
$$

Hence,

$$
\begin{aligned}
\operatorname{Var}\left(\mathbb{E}\left[T\left(Z, Z^{\prime}\right) \mid Z\right]\right) & \leq \frac{1}{2} \mathbb{E}\left[\sum_{k} \mathbb{E}_{Z^{\prime}}\left[T\left(Z^{k}, Z^{\prime}\right)-T\left(Z, Z^{\prime}\right) \mid Z, Z^{k}\right]^{2}\right] \\
& \leq \frac{1}{2} \sum_{k} \mathbb{E}\left[\left|T\left(Z^{k}, Z^{\prime}\right)-T\left(Z, Z^{\prime}\right)\right|^{2}\right]
\end{aligned}
$$


Recalling that

$$
T\left(Z, Z^{\prime}\right)=\frac{1}{2} \sum_{j=1}^{n} \sum_{\substack{A \subset[n] \\ j \notin A}} K_{n, A} \Delta_{j} f(Z) \Delta_{j} f\left(Z^{A}\right),
$$

we conclude that

$$
\begin{aligned}
\operatorname{Var}\left(\mathbb{E}\left[T\left(Z, Z^{\prime}\right) \mid Z\right]\right) \leq & \frac{1}{8} \sum_{k} \mathbb{E}\left[\left|\sum_{j=1}^{n} \sum_{\substack{A \subset[n] \\
j \notin A}} K_{n, A}\left(\Delta_{j} f(Z) \Delta_{j} f\left(Z^{A}\right)-\left(\Delta_{j} f(Z)\right)^{k}\left(\Delta_{j} f\left(Z^{A}\right)\right)^{k}\right)\right|^{2}\right] \\
\leq & \left.\sum_{k} \mathbb{E}\left[\left|\sum_{j=1}^{n} \sum_{\substack{A \subset[n] \\
j \notin A}} K_{n, A}\left(\Delta_{j} f(Z)-\left(\Delta_{j} f(Z)\right)^{k}\right) \Delta_{j} f\left(Z^{A}\right)\right|^{2}\right]\right] \\
& +\sum_{k} \mathbb{E}\left[\left|\sum_{j=1}^{n} \sum_{\substack{A \subset[n] \\
j \notin A}} K_{n, A}\left(\Delta_{j} f(Z)\right)^{k}\left(\Delta_{j} f\left(Z^{A}\right)-\left(\Delta_{j} f\left(Z^{A}\right)\right)^{k}\right)\right|^{2}\right] .
\end{aligned}
$$

Let us clarify the notation here. In the case $k \neq j$, we have

$$
\left(\Delta_{j} f(Z)\right)^{k}=f\left(Z_{1}, \ldots, Z_{j}^{\prime}, \ldots, Z_{k}^{\prime \prime}, \ldots, Z_{n}\right)-f\left(Z_{1}, \ldots, Z_{j}, \ldots, Z_{k}^{\prime \prime}, \ldots, Z_{n}\right)=\Delta_{j} f\left(Z^{k}, Z^{\prime}\right) .
$$

If $k=j$, then we have $\left(\Delta_{j} f(Z)\right)^{k}=\Delta_{j} f\left(Z^{k}, Z^{\prime}\right)=f\left(Z^{j}\right)-f\left(Z^{k}\right)$. Nevertheless, for all $j$ and $k$ we have

$$
\Delta_{j} f(Z)-\left(\Delta_{j} f(Z)\right)^{k}=-\Delta_{k} \Delta_{j} f(Z)=-\Delta_{k}\left(\Delta_{j} f\left(Z, Z^{\prime}\right)\right) .
$$

So, the first sum on the right side of (2.25) is

$$
\sum_{k} \mathbb{E}\left[\left|\sum_{j=1}^{n}\left(\Delta_{k}\left(\Delta_{j} f(Z)\right)\right) \overline{\Delta_{j} f\left(Z^{A}\right)}\right|^{2}\right],
$$

and the second sum is

$$
\sum_{k} \mathbb{E}\left[\left|\sum_{j=1}^{n}\left(\Delta_{j} f(Z)\right)^{k} \Delta_{k} \overline{\Delta_{j} f\left(Z^{A}\right)}\right|^{2}\right],
$$

where we have used the notation $\overline{\Delta_{j} f\left(Z^{A}\right)}$ to indicate averaging with respect to the set $A$. Specifically, if $S_{n, j}$ denotes the collection of all subsets $A \subset\{1, \ldots, n\}$ which do not contain the index $j$, and $H_{A}: S_{n, j} \rightarrow \mathbb{R}$, then

$$
\overline{H_{A}}=\sum_{\substack{A \subset[n] \\ j \notin A}} K_{n, A} H_{A}=\sum_{A \in S_{n, j}} K_{n, A} H_{A} .
$$

The weights $K_{n, A} \geq 0$ define a probability measure on $S_{n, j}: \sum_{A \in S_{n, j}} K_{n, A}=1$.

\section{Deterministic estimates for solutions of the elliptic equation}

In proving Theorem 1.1 we will make use of some regularity estimates - Cacciopoli's inequality and Meyers' estimate - that apply to solutions of elliptic PDEs. These estimates rely only on the uniform ellipticity assumption, not on the statistical structure of the coefficient $a(x)$ or on the periodicity. 


\section{Cacciopoli's inequality}

if $\bar{u}_{D}$ is the average of a function $u$ over a bounded domain $D$, then the Poincaré inequality is $\left\|u-\bar{u}_{D}\right\|_{L^{2}(D)} \leq C_{D}\|\nabla u\|_{L^{2}(D)}$. For solutions of elliptic equations, Cacciopoli's inequality gives the reverse inequality, enabling control of $\nabla u$ by $u$ itself. The basic estimate is:

Lemma 3.1 Let $d \geq 1$. There is a constant $K$ such that if $R>0$ and $u \in H^{1}\left(B_{R}\left(x_{0}\right)\right)$ is a weak solution to $-\nabla \cdot(a \nabla u)+\beta u=\nabla \cdot \xi$ for $x \in B_{R}\left(x_{0}\right)$, with $\xi \in\left(L^{2}\left(B_{R}\right)\right)^{d}$, then

$$
\int_{B_{\frac{R}{2}}\left(x_{0}\right)}|\nabla u|^{2} d x \leq K\left(\int_{B_{R}\left(x_{0}\right)}|\xi|^{2} d x+\frac{1}{R^{2}} \int_{B_{R}\left(x_{0}\right)}(u(x)-b)^{2} d x+\beta b^{2} R^{d}\right)
$$

holds for any constant $b \in \mathbb{R}$.

Lemma 3.1 and variants are a consequence of the following:

Lemma 3.2 Let $K_{1}=2 / a_{*}, K_{2}=\left(2 / a_{*}\right)+8\left(a^{*} / a_{*}\right)^{2}$, and $K_{3}=\left(2 / a_{*}\right)+2 /\left(a_{*}\right)^{2}$. Let $Q$ be $a$ bounded open subset of $\mathbb{R}^{d}$ with smooth boundary. If $\beta \geq 0$ and $u \in H^{1}(Q)$ is a weak solution to $-\nabla \cdot(a \nabla u)+\beta u=f+\nabla \cdot \xi$ for $x \in Q$, with $f \in L^{2}(Q)$ and $\xi \in\left(L^{2}(Q)\right)^{d}$, then

$$
\begin{aligned}
\int_{Q} \varphi^{2}|\nabla u|^{2} d x \leq & K_{1} \int_{Q} f(u-b) \varphi^{2} d x-K_{1} \beta \int_{Q} u(u-b) \varphi^{2} d x \\
& +K_{2} \int_{Q}|\nabla \varphi|^{2}(u-b)^{2} d x+K_{3} \int_{Q}|\xi|^{2} \varphi^{2} d x
\end{aligned}
$$

holds for any smooth function $\varphi \geq 0$ which vanishes on the boundary of $Q$, and any constant $b \in \mathbb{R}$.

For proofs of Lemma 3.1] and Lemma 3.2, see [29] (also [14], for example). The factor $R^{-2}$ in (3.29) comes from choosing a test function $\varphi$ in (3.30) with $|\nabla \varphi| \leq R^{-1}$. There is nothing special about the balls $B_{R}$ and $B_{2 R}$ in Lemma 3.1; for other nested domains whose boundaries are separated by distance $R$, a similar bound follows directly from Lemma 3.2 .

\section{Meyers' Estimate}

We also will make use of a well-known regularity estimate of Meyers [26] which shows that if $u \in H_{l o c}^{1}$ satisfies $-\nabla \cdot(a \nabla u)+\beta u=0$, then $\nabla u \in L_{l o c}^{p}$ for some $p>2$. Moreover, $\nabla u$ may be bounded as follows:

Lemma 3.3 There is a constant $p^{*}>2$, depending on $d$ and $a^{*} / a_{*}$, such that the following holds for all $p \in\left[2, p^{*}\right]$ : there is $C$ such that if $R>0$ and $u \in H^{1}\left(B_{4 R}(y)\right)$ satisfies $-\nabla \cdot(a \nabla u)+\beta u=0$ in $B_{4 R}(y)$, then

$$
\left(f_{B_{R}}|\nabla u|^{p} d x\right)^{1 / p} \leq C R^{-1}\left(f_{B_{4 R}} u^{2} d x\right)^{1 / 2} .
$$

Proof of Lemma 3.3; This is a consequence of Theorem 2 of [26] and Lemma 3.2. Since $u$ satisfies $-\nabla \cdot a \nabla u=h$ with $h=-\beta u$, we may apply Theorem 2 of Meyers' [26] to $u$ (with $p_{1}=2, r=2$ ), to conclude that for $p>2$ sufficiently small,

$$
\begin{aligned}
\left(f_{B_{R}}|\nabla u|^{p}\right)^{1 / p} & \leq C R^{-1}\left(f_{B_{2 R}}|u|^{2}\right)^{1 / 2}+C R\left(f_{B_{2 R}}|h|^{2} d x\right)^{1 / 2} \\
& =C R^{-1}\left(f_{B_{2 R}}|u|^{2}\right)^{1 / 2}+C R^{-1}\left(R^{4} \beta^{2} f_{B_{2 R}}|u|^{2} d x\right)^{1 / 2} .
\end{aligned}
$$


Now we estimate the last term in (3.31). Let $\varphi: \mathbb{R}^{d} \rightarrow[0,1]$ be a smooth function supported in $B_{3 R}(y)$ and satisfying $\varphi(x)=1$ for all $x \in B_{2 R}(y)$ and satisfying $|\nabla \varphi| \leq C / R$. Applying Lemma 3.2 with this function $\varphi$, with $b=0$ and with $Q=B_{3 R}(y)$, we conclude that

$$
\beta \int_{B_{2 R}} u^{2} d x \leq \beta \int_{B_{3 R}} u^{2} \varphi^{2} d x \leq C \int_{B_{3 R}} u^{2}|\nabla \varphi|^{2} d x \leq C R^{-2} \int_{B_{3 R}} u^{2} d x .
$$

Now we apply Lemma 3.2 once more, this time in $Q=B_{4 R}$, using a function $\varphi: \mathbb{R}^{d} \rightarrow[0,1]$ supported in $B_{4 R}(y)$ and satisfying $\varphi=1$ in $B_{3 R}(y)$ and $|\nabla \varphi| \leq C / R$. We conclude

$$
\beta \int_{B_{3 R}} u^{2} d x \leq \beta \int_{B_{4 R}} u^{2} \varphi^{2} d x \leq C \int_{B_{4 R}} u^{2}|\nabla \varphi|^{2} d x \leq C R^{-2} \int_{B_{4 R}} u^{2} d x .
$$

Combining (3.32) and (3.33) we obtain

$$
R^{4} \beta^{2} f_{B_{2 R}} u^{2} d x \leq C f_{B_{4 R}} u^{2} d x
$$

This combined with (3.31) implies the result.

\section{Application to the elliptic problem}

In this section we prove Theorem 1.1 by applying Theorem 2.4 to the random variable $f(Z)=$ $\Gamma_{L, \beta}(Z)$ defined by (1.2). In this case, the indices $j$ in Theorem 2.4 now run over the set $\mathbb{Z}^{d} \cap D_{L}$. The first step is to compute and estimate the terms $\Delta_{j} \Gamma$ and $\Delta_{k} \Delta_{j} \Gamma$ which appear in the sums (2.26) and (2.27).

\section{Estimating $\Delta_{j} \Gamma$ and $\Delta_{k} \Delta_{j} \Gamma$}

We will make use of the following chain rule and product rule for discrete differences:

$$
\Delta_{j}\left(f(Z)^{2}\right)=\left(\Delta_{j} f(Z)\right)\left(f\left(Z^{j}\right)+f(Z)\right)
$$

and

$$
\Delta_{j}(f(Z) g(Z))=\frac{1}{2}\left(\Delta_{j} f(Z)\right)\left(g\left(Z^{j}\right)+g(Z)\right)+\frac{1}{2}\left(f\left(Z^{j}\right)+f(Z)\right)\left(\Delta_{j} g(Z)\right) .
$$

Let us introduce the notation $a^{j}=a\left(x, Z^{j}\right), a^{k}=a\left(x, Z^{k}\right), \phi^{j}=\phi\left(x, Z^{j}\right), \phi^{k}=\phi\left(x, Z^{k}\right)$, according to (2.18) and (2.22). By the structural condition (1.7) observe that the functions

$$
\Delta_{j} a=a\left(x, Z^{j}\right)-a(x, Z), \quad \text { and } \quad \Delta_{k} a=a\left(x, Z^{k}\right)-a(x, Z)
$$

are supported on the sets $B_{\tau}(j)$ and $B_{\tau}(k)$ respectively. Furthermore, (1.7) implies that

$$
\Delta_{k} \Delta_{j} a=\Delta_{k}\left(a\left(x, Z^{j}\right)-a(x, Z)\right) \equiv 0, \quad \text { if } \operatorname{dist}(k, j) \geq 2 \tau .
$$

Lemma 4.1 There is a constant $C$ such that

$$
L^{d}\left|\Delta_{j} \Gamma(Z)\right| \leq C\left(\hat{\Phi}_{j}^{2}(Z)+\hat{\Phi}_{j}^{2}\left(Z^{j}\right)\right)
$$

holds for all $L>1, \beta \geq 0, j \in \mathbb{Z}^{d}$, where

$$
\hat{\Phi}_{j}(Z)=\left(\int_{B_{\tau}(j)}\left|\nabla \phi(x, Z)+e_{1}\right|^{2} d x\right)^{1 / 2} .
$$


Moreover, for any $q>1$, there is $C_{q}$ such that

$$
L^{q d} \mathbb{E}\left[\left|\Delta_{j} \Gamma(Z)\right|^{q}\right] \leq C_{q} \mathbb{E}\left[\left|\hat{\Phi}_{0}(Z)\right|^{2 q}\right] .
$$

and

$$
L^{q d} \mathbb{E}\left[\left|\overline{\Delta_{j} \Gamma\left(Z^{A}\right)}\right|^{q}\right] \leq C_{q} \mathbb{E}\left[\left|\hat{\Phi}_{0}(Z)\right|^{2 q}\right] .
$$

hold for all $L>1, \beta \geq 0, j \in \mathbb{Z}^{d}$.

Lemma 4.2 There is a constant $C$, independent of $L>1$ and $\beta \geq 0$ such that

$$
\begin{aligned}
L^{d}\left|\Delta_{k} \Delta_{j} \Gamma(Z)\right| \leq & C\left(\hat{\Phi}_{k}^{2}(Z)+\hat{\Phi}_{k}^{2}\left(Z^{j}\right)+\hat{\Phi}_{k}^{2}\left(Z^{k}\right)+\hat{\Phi}_{k}^{2}\left(Z^{j k}\right)\right) \\
& +C\left(\hat{\Phi}_{j}^{2}(Z)+\hat{\Phi}_{j}^{2}\left(Z^{j}\right)+\hat{\Phi}_{j}^{2}\left(Z^{k}\right)+\hat{\Phi}_{j}^{2}\left(Z^{j k}\right)\right)
\end{aligned}
$$

hold for all $k, j \in \mathbb{Z}^{d}$. Moreover,

$$
\begin{aligned}
L^{2 d}\left|\Delta_{k} \Delta_{j} \Gamma(Z)\right|^{2} \leq C( & \left.\hat{\Phi}_{j}^{2}(Z)+\hat{\Phi}_{j}^{2}\left(Z^{j}\right)+\hat{\Phi}_{j}^{2}\left(Z^{k}\right)+\hat{\Phi}_{j}^{2}\left(Z^{j k}\right)\right) \\
& \times \int_{B_{\tau}(j)}\left|\nabla \Delta_{k} \phi\right|^{2}+\left|\nabla \Delta_{k} \phi^{j}\right|^{2} d x
\end{aligned}
$$

holds for all $j, k$ with $\operatorname{dist}(k, j) \geq 2 \tau$.

Proof of Lemma 4.1; Using (4.34) and (4.35) and the symmetry of $a$ we compute:

$$
\begin{aligned}
L^{d} \Delta_{j} \Gamma(Z)= & \frac{1}{2} \int_{D_{L}}\left(\nabla \phi^{j}+e_{1}\right) \cdot\left(\Delta_{j} a\right)\left(\nabla \phi^{j}+e_{1}\right)+\left(\nabla \phi+e_{1}\right) \cdot\left(\Delta_{j} a\right)\left(\nabla \phi+e_{1}\right) d x \\
& +\int_{D_{L}} \frac{1}{2}\left(\nabla \Delta_{j} \phi\right) \cdot\left(a^{j}+a\right)\left(\nabla \phi^{j}+\nabla \phi+2 e_{1}\right) d x+\beta \int_{D_{L}}\left(\Delta_{j} \phi\right)\left(\phi^{j}+\phi\right) d x .
\end{aligned}
$$

Due to (1.10), we have

$$
\begin{aligned}
& \int_{D_{L}}\left(\nabla \Delta_{j} \phi\right) \cdot a^{j}\left(\nabla \phi^{j}+e_{1}\right)+\beta\left(\Delta_{j} \phi\right) \phi^{j} d x=0, \\
& \int_{D_{L}}\left(\nabla \Delta_{j} \phi\right) \cdot a\left(\nabla \phi+e_{1}\right)+\beta\left(\Delta_{j} \phi\right) \phi d x=0 .
\end{aligned}
$$

Using that observation we simplify (4.41) to

$$
\begin{aligned}
L^{d} \Delta_{j} \Gamma(Z)= & \frac{1}{2} \int_{D_{L}}\left(\nabla \phi^{j}+e_{1}\right) \cdot\left(\Delta_{j} a\right)\left(\nabla \phi^{j}+e_{1}\right)+\left(\nabla \phi+e_{1}\right) \cdot\left(\Delta_{j} a\right)\left(\nabla \phi+e_{1}\right) d x \\
& +\frac{1}{2} \int_{D_{L}} \nabla \Delta_{j} \phi \cdot\left(\Delta_{j} a\right)\left(\nabla \phi+e_{1}\right) d x-\frac{1}{2} \int_{D_{L}} \nabla \Delta_{j} \phi \cdot\left(\Delta_{j} a\right)\left(\nabla \phi^{j}+e_{1}\right) d x \\
= & \frac{1}{2} \int_{D_{L}}\left(\nabla \phi^{j}+e_{1}\right) \cdot\left(\Delta_{j} a\right)\left(\nabla \phi^{j}+e_{1}\right)+\left(\nabla \phi+e_{1}\right) \cdot\left(\Delta_{j} a\right)\left(\nabla \phi+e_{1}\right) d x \\
& -\frac{1}{2} \int_{D_{L}} \nabla \Delta_{j} \phi \cdot\left(\Delta_{j} a\right) \nabla \Delta_{j} \phi d x \\
= & \int_{D_{L}}\left(\nabla \phi^{j}+e_{1}\right) \cdot\left(\Delta_{j} a\right)\left(\nabla \phi+e_{1}\right) d x .
\end{aligned}
$$


Because $\Delta_{j} a=a\left(x, Z^{j}\right)-a(x, Z)$ vanishes outside $B_{\tau}(j)$ (by (1.7) $)$, we then infer that

$$
L^{d}\left|\Delta_{j} \Gamma(Z)\right| \leq C \int_{B_{\tau}(j)}\left|\nabla \phi^{j}+e_{1}\right|^{2} d x+C \int_{B_{\tau}(j)}\left|\nabla \phi+e_{1}\right|^{2} d x=C\left(\hat{\Phi}_{j}^{2}(Z)+\hat{\Phi}_{j}^{2}\left(Z^{j}\right)\right) .
$$

Since $\phi$ is stationary with respect to integer shifts and because $Z$ and $Z^{j}$ have the same law, the random variables $\hat{\Phi}_{j}(Z), \hat{\Phi}_{j}\left(Z^{j}\right)$, and $\hat{\Phi}_{0}(Z)$ are identically distributed. Therefore, for any $q>1$ there is a constant $C_{q}$ such that

$$
L^{q d} \mathbb{E}\left[\left|\Delta_{j} \Gamma(Z)\right|^{q}\right] \leq C_{q} \mathbb{E}\left[\left|\hat{\Phi}_{j}(Z)\right|^{2 q}\right]+C_{q} \mathbb{E}\left[\left|\hat{\Phi}_{j}\left(Z^{j}\right)\right|^{2 q}\right]=2 C_{q} \mathbb{E}\left[\left|\hat{\Phi}_{0}(Z)\right|^{2 q}\right],
$$

which is (4.37).

Now we prove (4.38). Jensen's inequality implies

$$
\left|\overline{\Delta_{j} \Gamma\left(Z^{A}\right)}\right|^{q}=\left|\sum_{\substack{A \subset[n] \\ j \notin A}} K_{n, A} \Delta_{j} \Gamma\left(Z^{A}\right)\right|^{q} \leq \sum_{\substack{A \subset[n] \\ j \notin A}} K_{n, A}\left|\Delta_{j} \Gamma\left(Z^{A}\right)\right|^{q} .
$$

Therefore from (4.37) we obtain

$$
L^{q d} \mathbb{E}\left[\left|\overline{\Delta_{j} \Gamma\left(Z^{A}\right)}\right|^{q}\right] \leq L^{q d} \sum_{\substack{A \subset[n] \\ j \notin A}} K_{n, A} \mathbb{E}\left[\left|\Delta_{j} \Gamma\left(Z^{A}\right)\right|^{q}\right] \leq C \mathbb{E}\left[\left|\hat{\Phi}_{0}(Z)\right|^{2 q}\right] .
$$

Proof of Lemma 4.2; Starting from (4.42) and using (4.34) and (4.35) we compute

$$
\begin{aligned}
L^{d} \Delta_{k} \Delta_{j} \Gamma(Z)= & \frac{1}{2} \int_{D_{L}}\left(\nabla \phi^{j k}+e_{1}\right) \cdot\left(\Delta_{k} \Delta_{j} a\right)\left(\nabla \phi^{k}+e_{1}\right)+\left(\nabla \phi^{j}+e_{1}\right) \cdot\left(\Delta_{k} \Delta_{j} a\right)\left(\nabla \phi+e_{1}\right) d x \\
& +\frac{1}{4} \int_{D_{L}} \Delta_{k} \nabla \phi^{j} \cdot\left(\left(\Delta_{j} a\right)^{k}+\left(\Delta_{j} a\right)\right)\left(\nabla \phi^{k}+e_{1}+\nabla \phi+e_{1}\right) \\
& +\frac{1}{4} \int_{D_{L}} \Delta_{k} \nabla \phi \cdot\left(\left(\Delta_{j} a\right)^{k}+\left(\Delta_{j} a\right)\right)\left(\nabla \phi^{j k}+e_{1}+\nabla \phi^{j}+e_{1}\right) .
\end{aligned}
$$

The matrices $\Delta_{j} a$ and $\Delta_{k} a$ are zero outside $B_{\tau}(j)$ and $B_{\tau}(k)$, respectively. Also, $\Delta_{k} \nabla \phi=\Delta_{k}(\nabla \phi+$ $\left.e_{1}\right)=\left(\nabla \phi^{k}+e_{1}\right)-\left(\nabla \phi+e_{1}\right)$. Therefore, by the Cauchy-Schwarz inequality we obtain

$$
\begin{aligned}
L^{d}\left|\Delta_{k} \Delta_{j} \Gamma(Z)\right| \leq & C\left(\hat{\Phi}_{k}^{2}(Z)+\hat{\Phi}_{k}^{2}\left(Z^{j}\right)+\hat{\Phi}_{k}^{2}\left(Z^{k}\right)+\hat{\Phi}_{k}^{2}\left(Z^{j k}\right)\right) \\
& +C\left(\hat{\Phi}_{j}^{2}(Z)+\hat{\Phi}_{j}^{2}\left(Z^{j}\right)+\hat{\Phi}_{j}^{2}\left(Z^{k}\right)+\hat{\Phi}_{j}^{2}\left(Z^{j k}\right)\right) .
\end{aligned}
$$

for all $j, k \in \mathbb{Z}^{d}$. This is (4.39).

If $\operatorname{dist}(k, j) \geq 2 \tau$ then $\left(\Delta_{k} \Delta_{j} a\right) \equiv 0$ and $\left(\Delta_{j} a\right)^{k}=\Delta_{j} a$, by (4.36). So, in this case we have

$$
\begin{aligned}
L^{d} \Delta_{k} \Delta_{j} \Gamma(Z)= & \frac{1}{2} \int_{D_{L}}\left(\Delta_{k} \nabla \phi^{j}\right) \cdot\left(\Delta_{j} a\right)\left(\nabla \phi^{k}+e_{1}+\nabla \phi+e_{1}\right) \\
& +\frac{1}{2} \int_{D_{L}}\left(\Delta_{k} \nabla \phi\right) \cdot\left(\Delta_{j} a\right)\left(\nabla \phi^{j k}+e_{1}+\nabla \phi^{j}+e_{1}\right) .
\end{aligned}
$$


Applying Cauchy-Schwarz to this, using the fact that $\Delta_{j} a$ is supported on $B_{\tau}(j)$, we obtain

$$
\begin{aligned}
L^{2 d}\left|\Delta_{k} \Delta_{j} \Gamma(Z)\right|^{2} \leq & C \int_{B_{\tau}(j)}\left|\nabla\left(\Delta_{k} \phi^{j}\right)\right|^{2} d x\left(\int_{B_{\tau}(j)}\left|\nabla \phi^{k}+e_{1}\right|^{2} d x+\int_{B_{\tau}(j)}\left|\nabla \phi+e_{1}\right|^{2} d x\right) \\
& +C \int_{B_{\tau}(j)}\left|\nabla\left(\Delta_{k} \phi\right)\right|^{2} d x\left(\int_{B_{\tau}(j)}\left|\nabla \phi^{j k}+e_{1}\right|^{2} d x+\int_{B_{\tau}(j)}\left|\nabla \phi^{j}+e_{1}\right|^{2} d x\right)
\end{aligned}
$$

if $\operatorname{dist}(k, j) \geq 2 \tau$. The implies (4.40).

\section{Relation to the periodic Green's function}

The function $w_{k}=\Delta_{k} \phi=\phi^{k}-\phi \in H_{p e r}^{1}\left(D_{L}\right)$ which appears in Lemma 4.2 satisfies the equation

$$
-\nabla \cdot\left(a \nabla w_{k}\right)+\beta w_{k}=\nabla \cdot\left(\Delta_{k} a\right)\left(\nabla \phi^{k}+e_{1}\right),
$$

and the distribution on the right side of (4.46) is supported on $\overline{B_{\tau}(k)}$. Choosing $w_{k}$ itself as a test function for (4.46), we obtain the bound

$$
\int_{D_{L}}\left|\nabla w_{k}\right|^{2} d x \leq\left(\frac{a^{*}}{a_{*}}\right)^{2} \int_{B_{\tau}(k)}\left|\nabla \phi^{k}+e_{1}\right|^{2} d x=\left(\frac{a^{*}}{a_{*}}\right)^{2} \hat{\Phi}_{k}^{2}\left(Z^{k}\right) .
$$

Later it will be convenient to normalize the function $w_{k}=\Delta_{k} \phi$ by defining

$$
\tilde{w}_{k}=\hat{\Phi}_{k}\left(Z^{k}\right)^{-1} w_{k}=\left(\int_{B_{\tau}(k)}\left|\nabla \phi^{k}+e_{1}\right|^{2} d x\right)^{-1 / 2} w_{k} .
$$

The following estimate relates $w_{k}$ to the periodic Green's function, and it will enable us to control the decay of $\left|\nabla w_{k}\right|^{2}$ away from $B_{\tau}(k)$ (using Cacciopoli's inequality). This connection between the Green's function and quantities analogous to $\Delta_{k} \phi$ has been used in other works, as well (e.g. [28, 17, 16]).

Lemma 4.3 Let $d \geq 1$, and let $G=G(x, y, Z)$ be the periodic Green's function associated with the coefficient $a(x, Z)$ :

$$
-\nabla_{x} \cdot\left(a(x, Z) \nabla_{x} G\right)+\beta G=\delta_{y}(x)-\left|D_{L}\right|^{-1},
$$

normalized by $\int_{D_{L}} G(x, y) d x=0$ in the case $\beta=0$. There is a constant $C$ (depending only on $\left.d, a_{*}, a^{*}\right)$ such that for any $L>2$, any $k \in D_{L} \cap \mathbb{Z}^{d}$, and any open set $A \subset D_{L}$ with $\operatorname{dist}\left(A, B_{\tau}(k)\right)>$ 0 , we have

$$
\int_{A}\left(\Delta_{k} \phi\right)^{2} d y \leq C \hat{\Phi}_{k}^{2}\left(Z^{k}\right) \int_{y \in A} \int_{x \in B_{\tau}(k)}\left|\nabla_{x} G(x, y)\right|^{2} d x d y .
$$

with probability one.

Proof of Lemma 4.3; Let us define $\xi_{k}=\left(\Delta_{k} a\right)\left(\nabla \phi^{k}+e_{1}\right)$ which is supported in $B_{\tau}(k)$. Let $v \in H_{p e r}^{1}\left(D_{L}\right)$ satisfy

$$
-\nabla \cdot(a \nabla v)+\beta v=\Delta_{k} \phi \mathbb{I}_{A}(x)-\frac{1}{D_{L}} \int_{A} \Delta_{k} \phi(x) d x
$$


By using (4.46) and the fact that $\int_{D_{L}} \Delta_{k} \phi(x) d x=0$, we have

$$
\begin{aligned}
\int_{A}\left(\Delta_{k} \phi(x)\right)^{2} d x & =\int_{D_{L}}\left(\mathbb{I}_{A}(x) \Delta_{k} \phi(x)\right) \Delta_{k} \phi(x) d x \\
& =\int_{D_{L}} \nabla v \cdot a(x) \nabla\left(\Delta_{k} \phi\right)+\beta v\left(\Delta_{k} \phi\right) d x \\
& =-\int_{D_{L}} \xi_{k}(x) \cdot \nabla v(x) d x \leq\left(\int_{B_{\tau}(k)}\left|\xi_{k}\right|^{2}\right)^{1 / 2}\left(\int_{B_{\tau}(k)}|\nabla v|^{2}\right)^{1 / 2} .
\end{aligned}
$$

On the other hand,

$$
v(x)=\int_{A} G(x, y) \Delta_{k} \phi(y) d y, \quad \nabla v(x)=\int_{A} \nabla_{x} G(x, y) \Delta_{k} \phi(y) d y
$$

hold for almost every $x$ outside $A$. Therefore, by Cauchy-Schwarz we have

$$
|\nabla v(x)|^{2} \leq \int_{A}\left|\nabla_{x} G(x, y)\right|^{2} d y \int_{A}\left(\Delta_{k} \phi(y)\right)^{2} d y
$$

for almost every $x$ in $B_{\tau}(j)$. Also, $\int_{B_{\tau}(k)}\left|\xi_{k}\right|^{2} d x \leq C_{2}^{2} \hat{\Phi}_{k}$, by (1.7). Combining this with (4.50) we obtain (4.49).

In view of Lemma 4.2 and Lemma 4.3 , we see that estimates of the Green's function will play an important role in estimating $\Delta_{k} \Delta_{j} \Gamma$. We will make use of the following bounds, proved later in Section 5. The first is a bound on the decay of $G(x, y)$ which is uniform with respect to the probability measure $\mathbb{P}$. The second, is a version of Lemma 2.9 in [17], and it is also uniform with respect to the probability measure $\mathbb{P}$. Recall the definitions (1.4) and (1.5) of $\operatorname{dist}(x, y)$ and $B_{r}(x)$.

Lemma 4.4 Let $d \geq 3$. There is a constant $C>0$, depending only on $d, a^{*}$, and $a_{*}$, such that

$$
|G(x, y)| \leq C \operatorname{dist}(x, y)^{2-d}
$$

holds for all $x, y \in D_{L}$ with $x \neq y$, all $L \geq 1$ and $\beta \geq 0$.

Lemma 4.5 Let $d=2$. There is a constant $C>0$, depending only on $d, a^{*}$, and $a_{*}$, such that for all $R>0, \beta \geq 0, L \geq 1$,

$$
\int_{B_{R}\left(x_{0}\right)}\left|\nabla_{x} G(x, y)\right|^{2} d x \leq C
$$

holds for all $x_{0} \in D_{L}$ and $y \in D_{L} \backslash \overline{B_{2 R}\left(x_{0}\right)}$.

\section{Proof of Theorem 1.1.}

Because of the stationarity assumption, moments of $\hat{\Phi}_{0}$ are controlled by the same moments of $\Phi_{0}$ : for any $q \geq 1$ there is a constant $C_{q}$ such that

$$
\mathbb{E}\left[\left|\hat{\Phi}_{0}\right|^{q}\right] \leq C_{q} \mathbb{E}\left[\left|\Phi_{0}\right|^{q}\right]
$$


for all $L \geq 1$ and $\beta \geq 0$. This is proved in Lemma 4.2 of [29], for example. Therefore, according to Lemma 4.1, we can bound the first term on the right side of (2.21) as

$$
\frac{1}{2 \sigma^{3}} \sum_{j \in D_{L}}\left\langle\left|\Delta_{j} \Gamma(Z)\right|^{3}\right\rangle \leq C \frac{L^{d}}{\sigma^{3}} L^{-3 d} \mathbb{E}\left[\left|\hat{\Phi}_{0}(Z)\right|^{6}\right] \leq C \frac{L^{d}}{\sigma^{3}} L^{-3 d} \mathbb{E}\left[\left|\Phi_{0}(Z)\right|^{6}\right] .
$$

As shown already, the term $\operatorname{Var}\left(\mathbb{E}\left[T\left(Z, Z^{\prime}\right) \mid Z\right]\right)$ in (2.21) is controlled by the sum of (2.26) and (2.27). We now focus on estimating (2.26). By Minkowsi's inequality we have

$$
\left\langle\left|\sum_{j \in D_{L}}\left(\Delta_{k} \Delta_{j} \Gamma(Z)\right) \overline{\Delta_{j} \Gamma\left(Z^{A}\right)}\right|^{2}\right\rangle \leq\left(\sum_{j \in D_{L}}\left\langle\left|\Delta_{k} \Delta_{j} \Gamma(Z) \overline{\Delta_{j} \Gamma\left(Z^{A}\right)}\right|^{2}\right\rangle^{1 / 2}\right)^{2} .
$$

It will be convenient to split up this sum over domains resembling dyadic annuli centered around the cube $Q_{k}=k+[0,1)^{d}$. Let $N$ denote the smallest integer such that $2^{N} \tau \geq L / 4$. Hence, $N=O(\log L)$ and $L / 4 \leq 2^{N} \tau \leq L / 2$. Then, let $A_{0}^{k}$ denote the union of cubes that are close to $Q_{k}$ :

$$
A_{0}^{k}=\left\{x \in D_{L} \mid x \in Q_{j}, \quad 0 \leq \operatorname{dist}\left(Q_{j}, Q_{k}\right) \leq 2 \tau\right\},
$$

and for $\ell=1,2, \ldots, N-1$ let $A_{\ell}^{k}$ denote the set

$$
A_{\ell}^{k}=\left\{x \in D_{L} \mid x \in Q_{j}, 2^{\ell} \tau<\operatorname{dist}\left(Q_{j}, Q_{k}\right) \leq 2^{\ell+1} \tau\right\} .
$$

Again, we use $\operatorname{dist}\left(Q_{j}, Q_{k}\right)$ to refer to distance on the torus $D_{L}$ (modulo $L \mathbb{Z}^{d}$ ) between sets $Q_{j}$ and $Q_{k}$. Finally, define $A_{N}^{k}$ by

$$
A_{N}^{k}=\left\{x \in D_{L} \mid x \in Q_{j}, 2^{N} \tau<\operatorname{dist}\left(Q_{j}, Q_{k}\right)\right\} .
$$

Each set $A_{\ell}^{k}$ is a union of cubes, and has Lebesgue measure $\left|A_{\ell}^{k}\right|=O\left(2^{\ell d}\right)$. Let $A_{+}^{k}=D_{L} \backslash A_{0}^{k}$. Observe that $j \in A_{\ell}^{k}$ if and only if $Q_{j} \subset A_{\ell}^{k}$. Similarly, $j \in A_{+}^{k}$ if and only if $Q_{j} \in A_{\ell}^{k}$ for some $\ell \geq 1$. Thus,

$$
D_{L}=A_{0}^{k} \cup A_{+}^{k}=\bigcup_{\ell=0}^{N} A_{\ell}^{k} .
$$

In this way, we write the sum appearing in (4.53) as:

$$
\begin{aligned}
\sum_{j \in D_{L}}\left\langle\left|\Delta_{k} \Delta_{j} \Gamma(Z) \overline{\Delta_{j} \Gamma\left(Z^{A}\right)}\right|^{2}\right\rangle^{1 / 2}= & \sum_{j \in A_{0}^{k} \cup A_{1}^{k}}\left\langle\left|\Delta_{k} \Delta_{j} \Gamma(Z) \overline{\Delta_{j} \Gamma\left(Z^{A}\right)}\right|^{2}\right\rangle^{1 / 2} \\
& +\sum_{\ell=2}^{N} \sum_{j \in A_{\ell}^{k}}\left\langle\left|\Delta_{k} \Delta_{j} \Gamma(Z) \overline{\Delta_{j} \Gamma\left(Z^{A}\right)}\right|^{2}\right\rangle^{1 / 2} .
\end{aligned}
$$

We will bound the terms in (4.54) using the following Lemma. The first estimate will bound the terms with indices $j \in A_{0}^{k} \cup A_{1}^{k}$. The second estimate will be used for the other indices.

Lemma 4.6 For $p>1$ there is a constant $C_{p}$ such that if $k, j \in D_{L}$ with $\operatorname{dist}(k, j) \geq 2 \tau$ then

$$
L^{4 d}\left\langle\left|\Delta_{k} \Delta_{j} \Gamma(Z) \overline{\Delta_{j} \Gamma\left(Z^{A}\right)}\right|^{2}\right\rangle \leq C_{p}\left\langle\hat{\Phi}_{0}^{4 q}\right\rangle^{\frac{4}{2 q}}\left\langle\left(\int_{B_{\tau}(j)}\left|\nabla \tilde{w}_{k}\right|^{2} d x\right)^{p}\right\rangle^{1 / p}
$$

where $q=2 p /(p-1)$. Also, there is a constant $C$ such that

$$
L^{4 d}\left\langle\left|\Delta_{k} \Delta_{j} \Gamma(Z) \overline{\Delta_{j} \Gamma\left(Z^{A}\right)}\right|^{2}\right\rangle \leq C\left\langle\hat{\Phi}_{0}^{8}\right\rangle
$$

holds for all $j, k \in D_{L}, L>1, \beta \geq 0$. 
Proof: First we prove (4.56). By Lemma 4.2, we always have

$$
\begin{aligned}
L^{2 d}\left|\Delta_{k} \Delta_{j} \Gamma(Z)\right|^{2} \leq C( & \left.\hat{\Phi}_{j}^{2}(Z)+\hat{\Phi}_{j}^{2}\left(Z^{j}\right)+\hat{\Phi}_{j}^{2}\left(Z^{k}\right)+\hat{\Phi}_{j}^{2}\left(Z^{j k}\right)\right) \\
& \times\left(\hat{\Phi}_{k}^{2}(Z)+\hat{\Phi}_{k}^{2}\left(Z^{j}\right)+\hat{\Phi}_{k}^{2}\left(Z^{k}\right)+\hat{\Phi}_{k}^{2}\left(Z^{j k}\right)\right) .
\end{aligned}
$$

Moreover, the terms $\hat{\Phi}_{j}^{2}(Z), \hat{\Phi}_{j}^{2}\left(Z^{j}\right), \hat{\Phi}_{j}^{2}\left(Z^{k}\right), \hat{\Phi}_{j}^{2}\left(Z^{j k}\right), \hat{\Phi}_{k}^{2}(Z), \hat{\Phi}_{k}^{2}\left(Z^{j}\right), \hat{\Phi}_{k}^{2}\left(Z^{k}\right), \hat{\Phi}_{k}^{2}\left(Z^{j k}\right)$ are identically distributed, all having the same distribution as $\hat{\Phi}_{0}(Z)$. By Lemma 4.1, we know that

$$
L^{2 d}\left\langle\left|\overline{\Delta_{j} \Gamma\left(Z^{A}\right)}\right|^{2 q}\right\rangle^{1 / q} \leq C\left\langle\hat{\Phi}_{0}^{4 q}\right\rangle^{\frac{1}{q}} .
$$

Therefore, by applying the generalized Hölder inequality with $\frac{1}{4}+\frac{1}{4}+\frac{1}{2}=1$ we obtain

$$
\begin{aligned}
L^{2 d}\left\langle\left|\Delta_{k} \Delta_{j} \Gamma(Z) \overline{\Delta_{j} \Gamma\left(Z^{A}\right)}\right|^{2}\right\rangle \leq & C\left\langle\left(\hat{\Phi}_{j}^{2}(Z)+\hat{\Phi}_{j}^{2}\left(Z^{j}\right)+\hat{\Phi}_{j}^{2}\left(Z^{k}\right)+\hat{\Phi}_{j}^{2}\left(Z^{j k}\right)\right)\right. \\
& \left.\times\left(\hat{\Phi}_{k}^{2}(Z)+\hat{\Phi}_{k}^{2}\left(Z^{j}\right)+\hat{\Phi}_{k}^{2}\left(Z^{k}\right)+\hat{\Phi}_{k}^{2}\left(Z^{j k}\right)\right)\left|\overline{\Delta_{j} \Gamma\left(Z^{A}\right)}\right|^{2}\right\rangle \\
\leq & C\left\langle\hat{\Phi}_{0}^{8}\right\rangle^{\frac{1}{4}}\left\langle\hat{\Phi}_{0}^{8}\right\rangle^{\frac{1}{4}}\left\langle\left|\overline{\Delta_{j} \Gamma\left(Z^{A}\right)}\right|^{4}\right\rangle^{1 / 2} \\
\leq & C\left\langle\hat{\Phi}_{0}^{8}\right\rangle^{\frac{1}{4}}\left\langle\hat{\Phi}_{0}^{8}\right\rangle^{\frac{1}{4}} L^{-2 d}\left\langle\hat{\Phi}_{0}^{8}\right\rangle^{\frac{1}{2}} .
\end{aligned}
$$

This proves (4.56).

If $\operatorname{dist}(k, j) \geq 2 \tau$, Lemma 4.2 tells us that

$$
\begin{aligned}
L^{2 d}\left|\Delta_{k} \Delta_{j} \Gamma(Z)\right|^{2} \leq & C\left(\hat{\Phi}_{j}^{2}(Z)+\hat{\Phi}_{j}^{2}\left(Z^{j}\right)+\hat{\Phi}_{j}^{2}\left(Z^{k}\right)+\hat{\Phi}_{j}^{2}\left(Z^{j k}\right)\right) \\
& \times\left(\hat{\Phi}_{k}^{2}\left(Z^{k}\right) \int_{B_{\tau}(j)}\left|\nabla \tilde{w}_{k}\right|^{2} d x+\hat{\Phi}_{k}^{2}\left(Z^{j k}\right) \int_{B_{\tau}(j)}\left|\nabla \tilde{w}_{k}^{j}\right|^{2} d x\right),
\end{aligned}
$$

where

$$
\tilde{w}_{k}=\hat{\Phi}_{k}\left(Z^{k}\right)^{-1} \Delta_{k} \phi, \quad \tilde{w}_{k}^{j}=\hat{\Phi}_{k}\left(Z^{j k}\right)^{-1} \Delta_{k} \phi^{j} .
$$

Let $p>1$, let $q=2 p /(p-1)$ so that $\frac{1}{p}+\frac{1}{2 q}+\frac{1}{2 q}+\frac{1}{q}=1$. Then by (4.60) and the generalized Hölder inequality,

$$
\begin{aligned}
L^{2 d}\left\langle\left|\Delta_{k} \Delta_{j} \Gamma(Z) \overline{\Delta_{j} \Gamma\left(Z^{A}\right)}\right|^{2}\right\rangle & \\
\leq & C\left\langle\hat{\Phi}_{0}^{4 q}\right\rangle^{\frac{1}{2 q}}\left\langle\hat{\Phi}_{0}^{4 q}\right\rangle^{\frac{1}{2 q}}\left\langle\left(\int_{B_{\tau}(j)}\left|\nabla \tilde{w}_{k}\right|^{2} d x\right)^{p}\right\rangle^{1 / p}\left\langle\left|\overline{\Delta_{j} \Gamma\left(Z^{A}\right)}\right|^{2 q}\right\rangle^{1 / q} \\
& +C\left\langle\hat{\Phi}_{0}^{4 q}\right\rangle^{\frac{1}{2 q}}\left\langle\hat{\Phi}_{0}^{4 q}\right\rangle^{\frac{1}{2 q}}\left\langle\left(\int_{B_{\tau}(j)}\left|\nabla \tilde{w}_{k}^{j}\right|^{2} d x\right)^{p}\right\rangle^{1 / p}\left\langle\left|\overline{\Delta_{j} \Gamma\left(Z^{A}\right)}\right|^{2 q}\right\rangle^{1 / q} .
\end{aligned}
$$

If $j \neq k$, then $\Delta_{k} \phi$ and $\Delta_{k} \phi^{j}$ have the same distribution (since $\left(Z, Z^{k}\right)$ and $\left(Z^{j}, Z^{j k}\right)$ have the same joint distribution). Similarly, $\tilde{w}_{k}$ and $\tilde{w}_{k}^{j}$ must have the same distribution. Therefore,

$$
\left\langle\left(\int_{B_{\tau}(j)}\left|\nabla \tilde{w}_{k}^{j}\right|^{2} d x\right)^{p}\right\rangle^{1 / p}=\left\langle\left(\int_{B_{\tau}(j)}\left|\nabla \tilde{w}_{k}\right|^{2} d x\right)^{p}\right\rangle^{1 / p} .
$$

holds for all $j \neq k$. Combining this observation with (4.61) and (4.58) we obtain

$$
L^{4 d}\left\langle\left|\Delta_{k} \Delta_{j} \Gamma(Z) \overline{\Delta_{j} \Gamma\left(Z^{A}\right)}\right|^{2}\right\rangle \leq C\left\langle\hat{\Phi}_{0}^{4 q}\right\rangle^{\frac{4}{2 q}}\left\langle\left(\int_{B_{\tau}(j)}\left|\nabla \tilde{w}_{k}\right|^{2} d x\right)^{p}\right\rangle^{1 / p} .
$$


This completes the proof of Lemma 4.6.

Now we return to (4.54). For the first sum on the right side of (4.54), over indices $j$ near $k$, we apply Lemma 4.6 to obtain

$$
L^{2 d} \sum_{j \in A_{0}^{k} \cup A_{1}^{k}}\left\langle\left|\Delta_{k} \Delta_{j} \Gamma(Z) \overline{\Delta_{j} \Gamma\left(Z^{A}\right)}\right|^{2}\right\rangle^{1 / 2} \leq C\left(\left|A_{0}^{k}\right|+\left|A_{1}^{k}\right|\right)\left\langle\hat{\Phi}_{0}^{8}\right\rangle^{1 / 2} .
$$

For the second sum in (4.54), we apply Lemma 4.6 again to obtain

$$
L^{2 d} \sum_{\ell=2}^{N} \sum_{j \in A_{\ell}^{k}}\left\langle\left|\Delta_{k} \Delta_{j} \Gamma(Z) \overline{\Delta_{j} \Gamma\left(Z^{A}\right)}\right|^{2}\right\rangle^{1 / 2} \leq C\left\langle\hat{\Phi}_{0}^{4 q}\right\rangle^{\frac{1}{q}} \sum_{\ell=2}^{N} \sum_{j \in A_{\ell}^{k}}\left\langle\int_{B_{\tau}(j)}\left|\nabla \tilde{w}_{k}\right|^{2 p} d x\right\rangle^{\frac{1}{2 p}}
$$

From our definition of the annuli $A_{\ell}^{k}$ and $\tau>\sqrt{d}$, we see that

$$
\bigcup_{\substack{j \in A_{\ell}^{k} \\ \ell \geq 2}} B_{\tau}(j) \subset \bigcup_{\substack{j \in A_{\ell}^{k} \\ \ell \geq 1}} Q_{j}
$$

Furthermore, each ball $B_{\tau}(j)$ intersects only finitely many cubes $\left(O\left(\tau^{d}\right)\right.$ of them). So, the last integral in (4.64) can be replaced by an integral over $Q_{j}$, at the expense of a constant factor of order $O\left(\tau^{d}\right)$. Indeed, by Minkowski's inequality,

$$
\left\langle\int_{B_{\tau}(j)}\left|\nabla \tilde{w}_{k}\right|^{2 p} d x\right\rangle^{\frac{1}{2 p}} \leq \sum_{\substack{n \in D_{L} \\\left|B_{\tau}(j) \cap Q_{n}\right|>0}}\left\langle\int_{Q_{n}}\left|\nabla \tilde{w}_{k}\right|^{2 p} d x\right\rangle^{\frac{1}{2 p}}
$$

Therefore, (4.64) yields

$$
\begin{aligned}
L^{2 d} \sum_{\ell=2}^{N} \sum_{j \in A_{\ell}^{k}}\left\langle\left|\Delta_{k} \Delta_{j} \Gamma(Z) \overline{\Delta_{j} \Gamma\left(Z^{A}\right)}\right|^{2}\right\rangle^{1 / 2} & \leq C\left\langle\hat{\Phi}_{0}^{4 q}\right\rangle^{\frac{1}{q}} \sum_{\ell=2}^{N} \sum_{j \in A_{\ell}^{k}} \sum_{\substack{n \in D_{L} \\
\left|B_{\tau}(j) \cap Q_{n}\right|>0}}\left\langle\int_{Q_{n}}\left|\nabla \tilde{w}_{k}\right|^{2 p} d x\right\rangle^{\frac{1}{2 p}} \\
& \leq C\left\langle\hat{\Phi}_{0}^{4 q}\right\rangle^{\frac{1}{q}} \sum_{\ell=2}^{N} \sum_{j \in A_{\ell}^{k}} \sum_{\substack{n \in D_{L} \\
\operatorname{dist}\left(Q_{j}, Q_{n}\right)<\tau}}\left\langle\int_{Q_{n}}\left|\nabla \tilde{w}_{k}\right|^{2 p} d x\right\rangle^{\frac{1}{2 p}} \\
& \leq C \tau^{d}\left\langle\hat{\Phi}_{0}^{4 q}\right\rangle^{\frac{1}{q}} \sum_{\ell=1}^{N} \sum_{j \in A_{\ell}^{k}}\left\langle\int_{Q_{j}}\left|\nabla \tilde{w}_{k}\right|^{2 p} d x\right\rangle^{\frac{1}{2 p}} \\
& =C \tau^{d}\left\langle\hat{\Phi}_{0}^{4 q}\right\rangle^{\frac{1}{q}} \sum_{j \in A_{+}^{k}}\left\langle\int_{Q_{j}}\left|\nabla \tilde{w}_{k}\right|^{2 p} d x\right\rangle^{\frac{1}{2 p}} .
\end{aligned}
$$

We will now show that the last sum in (4.66) is $O(\log L)$.

Lemma 4.7 There is are constants $C>0$ and $p>1$ such that

$$
\sum_{j \in A_{+}^{k}}\left\langle\int_{Q_{j}}\left|\nabla \tilde{w}_{k}\right|^{2 p} d x\right\rangle^{\frac{1}{2 p}} \leq C \log L
$$


and

$$
\sum_{k \in A_{+}^{0}}\left\langle\int_{Q_{0}}\left|\nabla \tilde{w}_{k}\right|^{2 p} d x\right\rangle^{\frac{1}{2 p}} \leq C \log L
$$

for all $\beta \geq 0, L \geq 2, k \in D_{L} \cap \mathbb{Z}^{d}$, where $\tilde{w}_{k}$ is defined by 4 4.48).

Lemma 4.3 gives control of $\tilde{w}_{k}(y)$ in terms of $\nabla_{x} G(x=k, y)$. So, thinking heuristically, we expect that for $\operatorname{dist}(y, k) \gg 1, \nabla_{y} \tilde{w}_{k}(y)$ should decay like the mixed second derivative $\nabla_{y} \nabla_{x} G(k, y)$ of the Green function. So, if the constant-coefficient case is any guide, we should hope that $\nabla_{y} \tilde{w}_{k}(y)$ decays like $O\left(|y-k|^{-d}\right)$. Although we do not have uniform pointwise bounds on $\nabla_{y} \tilde{w}_{k}(y)$ of this sort, we still obtain (4.67), which is what we would obtain if we did have the uniform bound $\left|\nabla_{y} \tilde{w}_{k}(y)\right| \leq C(1+|y-k|)^{-d}$. In the proof below, the strategy is to use Cacciopoli's inequality to control $\nabla \tilde{w}_{k}$ by $\tilde{w}_{k}$, then Lemma 4.3 to control $\tilde{w}_{k}$ by $\nabla G$. Then we use stationarity and Cacciopoli's inequality again to control $\nabla G$ by $G$, for which we have uniform bounds in Lemma $4.4(d \geq 3)$. Cacciopoli's inequality is applied over a large domain (the dyadic annuli) to take advantage of the $R^{-2}$ factor in Lemma 3.1. In the context of the discrete version of this elliptic problem, a similar strategy is employed by Gloria and Otto [17] to control the decay of $\nabla_{x} G(x, y)$ in terms of the uniform decay of $G(x, y)$ and by Marahrens and Otto [25] to estimate moments $\left\langle\left|\nabla_{x} \nabla_{y} G(x, y)\right|^{2 p}\right\rangle^{1 /(2 p)} \leq O\left((1+|x-y|)^{-d}\right)$ of the discrete second derivative of $G$.

Proof of Lemma 4.7. By stationarity, we have

$$
\left\langle\int_{Q_{j}}\left|\nabla \tilde{w}_{k}\right|^{2 p} d x\right\rangle^{\frac{1}{2 p}}=\left\langle\int_{Q_{0}}\left|\nabla \tilde{w}_{k-j}\right|^{2 p} d x\right\rangle^{\frac{1}{2 p}},
$$

so the bound (4.68) is equivalent to (4.67). Therefore, we focus on proving (4.67).

The constant $p>1$ may be chosen so that $2 p \in\left(0, p^{*}\right)$, where $p^{*}>2$ is as in Lemma 3.3. We split the (4.67) over the diadic annuli, and apply Hölder's inequality with $2 p$ and $\frac{2 p}{2 p-1}$ :

$$
\begin{aligned}
\sum_{\ell=1}^{N} \sum_{j \in A_{\ell}^{k}}\left\langle\int_{Q_{j}}\left|\nabla \tilde{w}_{k}\right|^{2 p} d x\right\rangle^{\frac{1}{2 p}} & \leq \sum_{\ell=1}^{N}\left(\sum_{j \in A_{\ell}^{k}} 1^{2 p /(2 p-1)}\right)^{(2 p-1) /(2 p)}\left(\sum_{j \in A_{\ell}^{k}}\left\langle\int_{Q_{j}}\left|\nabla \tilde{w}_{k}\right|^{2 p} d x\right\rangle\right)^{1 / 2 p} \\
& =\sum_{\ell=1}^{N}\left|A_{\ell}^{k}\right|^{(2 p-1) /(2 p)}\left(\left\langle\int_{A_{\ell}^{k}}\left|\nabla \tilde{w}_{k}\right|^{2 p} d x\right\rangle\right)^{1 /(2 p)}
\end{aligned}
$$

For $\ell \geq 1$, let us use the notation $2 A_{\ell}^{k}$ to refer to the fattened annuli:

$$
2 A_{\ell}^{k}=\left\{x \in D_{L} \mid x \in Q_{j}, 2^{\ell-1} \tau<\operatorname{dist}\left(Q_{j}, Q_{k}\right) \leq 3 \cdot 2^{\ell} \tau\right\}, \quad \ell=1, \ldots, N-1,
$$

and

$$
2 A_{N}^{k}=\left\{x \in D_{L} \mid x \in Q_{j}, 2^{N-1} \tau<\operatorname{dist}\left(Q_{j}, Q_{k}\right)\right\} .
$$

Observe that $A_{\ell}^{k} \subset 2 A_{\ell}^{k}$ and $\operatorname{dist}\left(A_{\ell}^{k}, \partial\left(2 A_{\ell}^{k}\right)\right) \geq C 2^{\ell}$. Also, $\operatorname{dist}\left(2 A_{\ell}^{k}, B_{\tau}(k)\right)>0$. By Lemma 3.3 applied to $\tilde{w}_{k}$ and by Lemma 4.3 , we know that

$$
\begin{aligned}
\int_{A_{\ell}^{k}}\left|\nabla \tilde{w}_{k}\right|^{2 p} d x & \leq C\left(2^{\ell}\right)^{d-p(2+d)}\left(\int_{2 A_{\ell}^{k}}\left(\tilde{w}_{k}\right)^{2} d y\right)^{p} \\
& \leq C\left(2^{\ell}\right)^{d-p(2+d)}\left(\int_{y \in 2 A_{\ell}^{k}} \int_{x \in Q_{k}}\left|\nabla_{x} G(x, y)\right|^{2} d x d y\right)^{p} .
\end{aligned}
$$


Hence,

$$
\begin{aligned}
\sum_{j \in A_{+}^{k}}\left\langle\int_{Q_{j}}\left|\nabla \tilde{w}_{k}\right|^{2 p} d x\right\rangle^{\frac{1}{2 p}} \\
\quad \leq C \sum_{\ell=1}^{N}\left|A_{\ell}^{k}\right|^{(2 p-1) /(2 p)}\left(\left\langle\int_{A_{\ell}^{k}}\left|\nabla \tilde{w}_{k}\right|^{2 p} d x\right\rangle\right)^{1 /(2 p)} \\
\quad \leq C \sum_{\ell=1}^{N}\left(2^{\ell}\right)^{d(2 p-1) /(2 p)}\left(2^{\ell}\right)^{(d-p(2+d)) /(2 p)}\left\langle\int_{y \in 2 A_{\ell}^{k}} \int_{x \in Q_{k}}\left|\nabla_{x} G(x, y)\right|^{2} d x d y\right\rangle^{1 / 2} \\
\quad=C \sum_{\ell=1}^{N}\left(2^{\ell}\right)^{d / 2-1}\left\langle\int_{y \in 2 A_{\ell}^{k}} \int_{x \in Q_{k}}\left|\nabla_{x} G(x, y)\right|^{2} d x d y\right\rangle^{1 / 2} \cdot
\end{aligned}
$$

By stationarity we have

$$
\left\langle\int_{y \in Q_{j}} \int_{x \in Q_{k}}\left|\nabla_{x} G(x, y)\right|^{2} d x d y\right\rangle=\left\langle\int_{y \in Q_{0}} \int_{x \in Q_{k-j}}\left|\nabla_{x} G(x, y)\right|^{2} d x d y\right\rangle .
$$

Therefore,

$$
\left\langle\int_{y \in 2 A_{\ell}^{k}} \int_{x \in Q_{k}}\left|\nabla_{x} G(x, y)\right|^{2} d x d y\right\rangle=\left\langle\int_{y \in Q_{0}} \int_{x \in 2 A_{\ell}^{0}}\left|\nabla_{x} G(x, y)\right|^{2} d x d y\right\rangle .
$$

The point here is that the integral in $x$ is now over the annulus $A_{\ell}^{0}$ of diameter $O\left(2^{\ell}\right)$, rather than over the unit cube.

For $d \geq 3$, we combine (4.71) with Cacciopoli's inequality to $x \mapsto G(x, y)$. The result is:

$$
\begin{aligned}
\left\langle\int_{y \in 2 A_{\ell}^{k}} \int_{x \in Q_{k}}\left|\nabla_{x} G(x, y)\right|^{2} d x d y\right\rangle \leq & C\left(2^{\ell}\right)^{-2}\left\langle\int_{y \in Q_{0}} \int_{x \in 2 A_{\ell}^{0}}|G(x, y)|^{2} d x d y\right\rangle \\
& +C\left|D_{L}\right|^{-1}\left\langle\int_{y \in Q_{0}} \int_{x \in 2 A_{\ell}^{0}}|G(x, y)| d x d y\right\rangle .
\end{aligned}
$$

By Lemma 4.4, we have a uniform decay estimates for $|G(x, y)| \leq C \operatorname{dist}(x, y)^{2-d}$ for $d \geq 3$. Therefore,

$$
\left\langle\int_{y \in 2 A_{\ell}^{k}} \int_{x \in Q_{k}}\left|\nabla_{x} G(x, y)\right|^{2} d x d y\right\rangle \leq C\left(2^{\ell}\right)^{-2}\left(2^{\ell}\right)^{d+2(2-d)}+C L^{-d}\left(2^{\ell}\right)^{d}\left(2^{\ell}\right)^{2-d} \leq\left(2^{\ell}\right)^{2-d} .
$$

So, returning to (4.70), we obtain

$$
\sum_{j \in A_{+}^{k}}\left\langle\int_{Q_{j}}\left|\nabla \tilde{w}_{k}\right|^{2 p} d x\right\rangle^{1 / 2 p} \leq C \sum_{\ell=1}^{N}\left(2^{\ell}\right)^{d / 2-1}\left(2^{\ell}\right)^{1-d / 2}=O(\log L) .
$$

In the case $d=2$, we apply Lemma 4.5 directly to (4.71) and conclude

$$
\left\langle\int_{y \in 2 A_{\ell}^{k}} \int_{x \in Q_{k}}\left|\nabla_{x} G(x, y)\right|^{2} d x d y\right\rangle \leq C .
$$


So, returning to (4.70), we still obtain

$$
\sum_{j \in A_{+}^{k}}\left\langle\int_{Q_{j}}\left|\nabla \tilde{w}_{k}\right|^{2} d x\right\rangle^{1 / 2} \leq C \sum_{\ell=1}^{O(\log L)}\left(2^{\ell}\right)^{d / 2-1}=O(\log L)
$$

This completes the proof of Lemma 4.7 .

Now we combine (4.53), (4.54), 44.63), 4.66), (4.51) and Lemma 4.7 to conclude that

$$
\begin{aligned}
& \sum_{k} \mathbb{E}\left[\mid \sum_{j \in D_{L}}\left(\left.\Delta_{k} \Delta_{j} \Gamma(Z) \overline{\Delta_{j} \Gamma\left(Z^{A}\right)}\right|^{2}\right]\right. \\
& \quad \leq \sum_{k}\left(\sum_{j \in A_{0}^{k} \cup A_{1}^{k}}\left\langle\left|\Delta_{k} \Delta_{j} \Gamma(Z) \overline{\Delta_{j} \Gamma\left(Z^{A}\right)}\right|^{2}\right\rangle^{1 / 2}+\sum_{\ell=2}^{N} \sum_{j \in A_{\ell}^{k}}\left\langle\left|\Delta_{k} \Delta_{j} \Gamma(Z) \overline{\Delta_{j} \Gamma\left(Z^{A}\right)}\right|^{2}\right\rangle^{1 / 2}\right)^{2} \\
& \quad \leq \sum_{k}\left(C L^{-2 d}\left\langle\Phi_{0}^{8}\right\rangle^{1 / 2}+C\left\langle\Phi_{0}^{8 q}\right\rangle^{1 / 2 q} L^{-2 d} \log L\right)^{2} \\
& \leq C L^{-3 d}\left\langle\Phi_{0}^{8 q}\right\rangle^{1 / q}(\log L)^{2}
\end{aligned}
$$

holds for $d \geq 2$, for all $L \geq 2, \beta \geq 0$.

Finally, we estimate (2.27). By Minkowsi's inequality we have

$$
\mathbb{E}\left[\left|\sum_{j \in D_{L}}\left(\Delta_{j} \Gamma(Z)\right)^{k} \Delta_{k} \overline{\Delta_{j} \Gamma\left(Z^{A}\right)}\right|^{2}\right] \leq\left(\sum_{j \in D_{L}}\left\langle\left|\left(\Delta_{j} \Gamma(Z)\right)^{k} \Delta_{k} \overline{\Delta_{j} \Gamma\left(Z^{A}\right)}\right|^{2}\right\rangle^{1 / 2}\right)^{2} .
$$

Recall the notation (2.28) for the average with respect to sets $A$ not containing index $j$. In particular, the weights $K_{n, A}$ define a probability distribution over the index sets $A$ not containing $j$. By applying Jensen' inequality to (4.75) we obtain

$$
\begin{aligned}
& \left(\sum_{j \in D_{L}}\left\langle\left|\left(\Delta_{j} \Gamma(Z)\right)^{k} \Delta_{k} \overline{\Delta_{j} \Gamma\left(Z^{A}\right)}\right|^{2}\right\rangle^{1 / 2}\right)^{2}=\left(\sum_{j \in D_{L}}\left\langle\left.\overline{\left(\Delta_{j} \Gamma(Z)\right)^{k}\left(\Delta_{k}\left(\Delta_{j} \Gamma\right)\right)\left(Z^{A}, Z^{\prime}, Z^{\prime \prime}\right)}\right|^{2}\right\rangle^{1 / 2}\right)^{2} \\
& \leq\left(\sum_{j \in D_{L}}\left\langle\overline{\left.\left(\mid \Delta_{j} \Gamma(Z)\right)^{k}\left(\Delta_{k}\left(\Delta_{j} \Gamma\right)\right)\left(Z^{A}, Z^{\prime}, Z^{\prime \prime}\right)\right|^{2}}\right\rangle^{1 / 2}\right)^{2} \\
& =\left(\sum_{j \in D_{L}}\left\langle\left\langle\left|\left(\Delta_{j} \Gamma\right)^{k}\left(Z, Z^{\prime}, Z^{\prime \prime}\right)\left(\Delta_{k}\left(\Delta_{j} \Gamma\right)\right)\left(Z^{A}, Z^{\prime}, Z^{\prime \prime}\right)\right|^{2}\right\rangle\right\rangle^{1 / 2}\right)^{2}
\end{aligned}
$$

where we have introduced the notation

$$
\begin{aligned}
\left\langle\left\langle H_{A}\left(Z, Z^{\prime}, Z^{\prime \prime}\right)\right\rangle\right\rangle & =\mathbb{E}\left[\overline{H_{A}\left(Z, Z^{\prime}, Z^{\prime \prime}\right)}\right] \\
& =\mathbb{E}\left[\sum_{\substack{A \subset[n] \\
j \notin A}} K_{n, A} H_{A}\left(Z, Z^{\prime}, Z^{\prime \prime}\right)\right]=\sum_{\substack{A \subset[n] \\
j \notin A}} K_{n, A} \mathbb{E}\left[H_{A}\left(Z, Z^{\prime}, Z^{\prime \prime}\right)\right] .
\end{aligned}
$$

The rest proceeds exactly as in the proof of (4.74)), the only difference being the following modification of Lemma 4.6 . 
Lemma 4.8 For $p>1$ there is a constant $C_{p}$ such that if $k, j \in D_{L}$ with $|k-j|>2 \tau$ then

$$
L^{4 d}\left\langle\left\langle\left|\left(\Delta_{k} \Delta_{j} \Gamma\right)\left(Z^{A}, Z^{\prime}, Z^{\prime \prime}\right)\left(\Delta_{j} \Gamma\right)^{k}\left(Z, Z^{\prime}, Z^{\prime \prime}\right)\right|^{2}\right\rangle\right\rangle \leq C_{p}\left\langle\Phi_{0}^{4 q}\right\rangle^{\frac{4}{2 q}}\left\langle\left(\int_{B_{\tau}(j)}\left|\nabla \tilde{w}_{k}\right|^{2} d x\right)^{p}\right\rangle^{1 / p}
$$

where $q=2 p /(p-1)$. Also, there is a constant $C$ such that

$$
L^{4 d}\left\langle\left\langle\left|\left(\Delta_{k} \Delta_{j} \Gamma\right)\left(Z^{A}, Z^{\prime}, Z^{\prime \prime}\right)\left(\Delta_{j} \Gamma\right)^{k}\left(Z, Z^{\prime}, Z^{\prime \prime}\right)\right|^{2}\right\rangle\right\rangle \leq C\left\langle\Phi_{0}^{8}\right\rangle
$$

holds for all $j, k \in D_{L}, L>1, \beta \geq 0$.

Proof: The proof is almost identical to that of Lemma 4.6. We only need to observe that, for any pair of indices $j, k \in D_{L}$ and any set $A \subset D_{L} \cap \mathbb{Z}^{d}$, if $g\left(Z, Z^{\prime}, Z^{\prime \prime}\right)$ denotes any of the random variables $\hat{\Phi}_{j}^{2}(Z), \hat{\Phi}_{j}^{2}\left(Z^{j}\right), \hat{\Phi}_{j}^{2}\left(Z^{k}\right), \hat{\Phi}_{j}^{2}\left(Z^{j k}\right), \hat{\Phi}_{k}^{2}(Z), \hat{\Phi}_{k}^{2}\left(Z^{j}\right), \hat{\Phi}_{k}^{2}\left(Z^{k}\right)$, or $\hat{\Phi}_{k}^{2}\left(Z^{j k}\right)$, then $g\left(Z, Z^{\prime}, Z^{\prime \prime}\right)$ and $g\left(Z^{A}, Z^{\prime}, Z^{\prime \prime}\right)$ have the same distribution. In particular, for any power $p$,

$$
\left\langle\left\langle g\left(Z^{A}, Z^{\prime}, Z^{\prime \prime}\right)^{p}\right\rangle\right\rangle=\left\langle\left\langle g\left(Z, Z^{\prime}, Z^{\prime \prime}\right)^{p}\right\rangle\right\rangle=\left\langle g\left(Z, Z^{\prime}, Z^{\prime \prime}\right)^{p}\right\rangle=\left\langle\hat{\Phi}_{0}^{2 p}\right\rangle .
$$

Similarly, the random variables $\left(\Delta_{j} \Gamma\right)^{k}\left(Z, Z^{\prime}, Z^{\prime \prime}\right)$ and $\Delta_{j} \Gamma\left(Z, Z^{\prime}\right)$ have the same distribution. As before, by Lemma 4.2 and Lemma 4.1 , we have

$$
\begin{aligned}
L^{2 d}\left|\left(\Delta_{k} \Delta_{j} \Gamma\right)\left(Z, Z^{\prime}, Z^{\prime \prime}\right)\right|^{2} \leq C( & \left.\hat{\Phi}_{j}^{2}(Z)+\hat{\Phi}_{j}^{2}\left(Z^{j}\right)+\hat{\Phi}_{j}^{2}\left(Z^{k}\right)+\hat{\Phi}_{j}^{2}\left(Z^{j k}\right)\right) \\
& \times\left(\hat{\Phi}_{k}^{2}(Z)+\hat{\Phi}_{k}^{2}\left(Z^{j}\right)+\hat{\Phi}_{k}^{2}\left(Z^{k}\right)+\hat{\Phi}_{k}^{2}\left(Z^{j k}\right)\right)
\end{aligned}
$$

and

$$
L^{2 d}\left\langle\left|\left(\Delta_{j} \Gamma\right)^{k}\left(Z, Z^{\prime}, Z^{\prime \prime}\right)\right|^{2 q}\right\rangle^{1 / q}=L^{2 d}\left\langle\left|\Delta_{j} \Gamma\left(Z, Z^{\prime}\right)\right|^{2 q}\right\rangle^{1 / q} \leq C\left\langle\hat{\Phi}_{0}^{4 q}\right\rangle^{\frac{1}{q}} .
$$

Therefore, as in the proof of Lemma 4.6, by applying the generalized Hölder inequality and (4.79) we obtain

$$
\begin{aligned}
L^{2 d}\left\langle\left\langle\left|\left(\Delta_{k} \Delta_{j} \Gamma\right)\left(Z^{A}, Z^{\prime}, Z^{\prime \prime}\right)\left(\Delta_{j} \Gamma\right)^{k}\left(Z, Z^{\prime}, Z^{\prime \prime}\right)\right|^{2}\right\rangle\right\rangle & \leq C\left\langle\hat{\Phi}_{0}^{8}\right\rangle^{\frac{1}{4}}\left\langle\hat{\Phi}_{0}^{8}\right\rangle^{\frac{1}{4}}\left\langle\left|\left(\Delta_{j} \Gamma\right)^{k}\right|^{4}\right\rangle^{1 / 2} \\
& \leq C\left\langle\hat{\Phi}_{0}^{8}\right\rangle^{\frac{1}{4}}\left\langle\hat{\Phi}_{0}^{8}\right\rangle^{\frac{1}{4}} L^{-2 d}\left\langle\hat{\Phi}_{0}^{8}\right\rangle^{\frac{1}{2}} .
\end{aligned}
$$

This and (4.51) imply (4.78).

If $\operatorname{dist}(k, j)>2 \tau$, Lemma 4.2 tells us that

$$
\begin{aligned}
L^{2 d}\left|\Delta_{k} \Delta_{j} \Gamma(Z)\right|^{2} \leq & C\left(\hat{\Phi}_{j}^{2}(Z)+\hat{\Phi}_{j}^{2}\left(Z^{j}\right)+\hat{\Phi}_{j}^{2}\left(Z^{k}\right)+\hat{\Phi}_{j}^{2}\left(Z^{j k}\right)\right) \\
& \times\left(\hat{\Phi}_{k}^{2}\left(Z^{k}\right) \int_{B_{\tau}(j)}\left|\nabla \tilde{w}_{k}\right|^{2} d x+\Phi_{k}^{2}\left(Z^{j k}\right) \int_{B_{\tau}(j)}\left|\nabla \tilde{w}_{k}^{j}\right|^{2} d x\right),
\end{aligned}
$$

where

$$
\tilde{w}_{k}=\hat{\Phi}_{k}\left(Z^{k}\right)^{-1} \Delta_{k} \phi, \quad \tilde{w}_{k}^{j}=\hat{\Phi}_{k}\left(Z^{j k}\right)^{-1} \Delta_{k} \phi^{j} .
$$

Let $p>1$, let $q=2 p /(p-1)$ so that $\frac{1}{p}+\frac{1}{2 q}+\frac{1}{2 q}+\frac{1}{q}=1$. Then by (4.81) and the generalized Hölder inquality,

$$
\begin{aligned}
& L^{2 d}\left\langle\left\langle\left|\left(\Delta_{k} \Delta_{j} \Gamma\right)\left(Z^{A}, Z^{\prime}, Z^{\prime \prime}\right)\left(\Delta_{j} \Gamma\right)^{k}\left(Z, Z^{\prime}, Z^{\prime \prime}\right)\right|^{2}\right\rangle\right\rangle \\
& \leq C\left\langle\hat{\Phi}_{0}^{4 q}\right\rangle^{\frac{1}{2 q}}\left\langle\hat{\Phi}_{0}^{4 q}\right\rangle^{\frac{1}{2 q}}\left\langle\left\langle g_{j k}\left(Z^{A}, Z_{k}^{\prime \prime}\right)^{p}\right\rangle\right\rangle^{1 / p}\left\langle\left|\left(\Delta_{j} \Gamma\right)^{k}\left(Z, Z^{\prime}, Z^{\prime \prime}\right)\right|^{2 q}\right\rangle^{1 / q} \\
&+C\left\langle\hat{\Phi}_{0}^{4 q}\right\rangle^{\frac{1}{2 q}}\left\langle\hat{\Phi}_{0}^{4 q}\right\rangle^{\frac{1}{2 q}}\left\langle\left\langle g_{j k}\left(Z^{j \cup A}, Z_{k}^{\prime \prime}\right)^{p}\right\rangle\right\rangle^{1 / p}\left\langle\left|\left(\Delta_{j} \Gamma\right)^{k}\left(Z, Z^{\prime}, Z^{\prime \prime}\right)\right|^{2 q}\right\rangle^{1 / q}
\end{aligned}
$$


where

$$
g_{j k}\left(Z, Z_{k}^{\prime \prime}\right)=\int_{B_{\tau}(j)}\left|\nabla_{x} \tilde{w}_{k}\left(x, Z, Z_{k}^{\prime \prime}\right)\right|^{2} d x .
$$

On the other hand, $g_{j k}\left(Z, Z_{k}^{\prime \prime}\right)$ and $g_{j k}\left(Z^{A}, Z_{k}^{\prime \prime}\right)$ and $g_{j k}\left(Z^{j \cup A}, Z_{k}^{\prime \prime}\right)$ all have the same distribution. Hence

$$
\left\langle\left\langle g_{j k}\left(Z^{j \cup A}, Z_{k}^{\prime \prime}\right)^{p}\right\rangle\right\rangle^{1 / p}=\left\langle\left\langle g_{j k}\left(Z^{A}, Z_{k}^{\prime \prime}\right)^{p}\right\rangle\right\rangle^{1 / p}=\left\langle\left(\int_{B_{\tau}(j)}\left|\nabla \tilde{w}_{k}\right|^{2} d x\right)^{p}\right\rangle^{1 / p} .
$$

We conclude that

$$
\begin{aligned}
& L^{2 d}\left\langle\left\langle\left|\left(\Delta_{k} \Delta_{j} \Gamma\right)\left(Z^{A}, Z^{\prime}, Z^{\prime \prime}\right)\left(\Delta_{j} \Gamma\right)^{k}\left(Z, Z^{\prime}, Z^{\prime \prime}\right)\right|^{2}\right\rangle\right\rangle \\
& \quad \leq C\left\langle\hat{\Phi}_{0}^{4 q}\right\rangle^{\frac{1}{2 q}}\left\langle\hat{\Phi}_{0}^{4 q}\right\rangle^{\frac{1}{2 q}}\left\langle\left(\int_{B_{\tau}(j)}\left|\nabla \tilde{w}_{k}\right|^{2} d x\right)^{p}\right\rangle^{1 / p}\left\langle\left|\Delta_{j} \Gamma(Z)\right|^{2 q}\right\rangle^{1 / q} \\
& \quad \leq C\left\langle\hat{\Phi}_{0}^{4 q}\right\rangle^{\frac{1}{2 q}}\left\langle\hat{\Phi}_{0}^{4 q}\right\rangle^{\frac{1}{2 q}}\left\langle\left(\int_{B_{\tau}(j)}\left|\nabla \tilde{w}_{k}\right|^{2} d x\right)^{p}\right\rangle^{1 / p} L^{-2 d}\left\langle\Phi_{0}^{4 q}\right\rangle^{1 / q}
\end{aligned}
$$

which implies (4.77).

With this modification of Lemma 4.6, we proceed exactly as in the proof of (4.74) to obtain the bound

$$
\left.\left.\sum_{k} \mathbb{E}\left[\mid \sum_{j \in D_{L}}\left(\Delta_{j} \Gamma\right)^{k}(Z)\right)^{k} \Delta_{k} \overline{\Delta_{j} \Gamma\left(Z^{A}\right)}\right|^{2}\right] \leq C\left\langle\Phi_{0}^{8 q}\right\rangle^{1 / q} L^{d} L^{-4 d}(\log L)^{2} .
$$

By combining Theorem 2.4 with (4.52), (4.74), and (4.84) we conclude that

$$
d_{\mathcal{W}}\left(\frac{\Gamma_{L, \beta}-m_{L, \beta}}{\sigma_{L, \beta}}, Z\right) \leq C \frac{L^{-2 d}}{\sigma^{3}} \mathbb{E}\left[\Phi_{0}^{6}\right]+C \frac{L^{-3 d / 2} \log (L)}{\sigma^{2}} \mathbb{E}\left[\Phi_{0}^{8 q}\right]^{\frac{1}{2 q}} .
$$

for all $d \geq 2$. The exponent $q>2$ is the Hölder conjugate of $p$, where $2 p$ is the exponent from Meyers' estimate. This concludes the proof of Theorem 1.1.

\section{Estimates for the periodic Green's function}

\section{$d \geq 3:$ Proof of Lemma 4.4}

Here we follow ideas used to prove a uniform decay estimate for Green's functions in $\mathbb{R}^{d}$, as in Theorem 1.1 of [20] and Lemma 2.8 of [17]; the difference here is the periodicity, so we include a proof for completeness. Let $y \in D_{L}$ and let $u(x)=G(x, y)$ be the periodic Green's function, which satisfies

$$
-\nabla \cdot(a \nabla u)+\beta u=\delta_{y}-\left|D_{L}\right|^{-1}, \quad x \in D_{L} .
$$

in the weak sense. Suppose that $u$ also satisfies

$$
\left|\left\{x \in D_{L} \mid u(x)>0\right\}\right| \leq \frac{1}{2}\left|D_{L}\right| .
$$

(If this is not the case, then we could apply the same argument to the function $-u$ instead.) Then, for any $k>0$, the function $u_{k}(x)=\max (0, \min (u, k))$ satisfies

$$
\left|\left\{x \in D_{L} \mid u_{k}(x) \neq 0\right\}\right|=\left|\left\{x \in D_{L} \mid u(x)>0\right\}\right| \leq \frac{1}{2}\left|D_{L}\right|
$$


Since $\left\|u_{k}\right\|_{\infty} \leq k$, we observe that $u_{k}$ satisfies

$$
\int_{D_{L}} \nabla u_{k} \cdot a \nabla u_{k} d x=\int_{D_{L}} \nabla u \cdot a \nabla u_{k} d x \leq-\beta \int_{D_{L}} u u_{k} d x+2 k \leq 2 k .
$$

Therefore,

$$
\int_{D_{L}}\left|\nabla u_{k}\right|^{2} d x \leq 2 k / a_{*}
$$

Considering (5.88), we know there is a constant $C$, independent of $k, L$ and $\beta$, such that

$$
\left(\int_{D_{L}}\left|u_{k}\right|^{q} d x\right)^{1 / q} \leq C\left(\int_{D_{L}}\left|\nabla u_{k}\right|^{2} d x\right)^{1 / 2}
$$

where $q=2 d /(d-2)$ is the critical Sobolev exponent. By scaling, this is a consequence of the Sobolev imbedding theorem and the Poincaré inequality for functions $v \in H_{p e r}^{1}\left(D_{1}\right)$ which also satisfy $\left|\left\{x \in D_{1} \mid v(x)=0\right\}\right| \geq 1 / 2$ (for example, see Lemma 4.8 of [21]). By applying Chebychev's inequality, then (5.90) and (5.89), we obtain the estimate

$$
\left|\left\{x \in D_{L} \mid u(x) \geq k\right\}\right|=\left|\left\{x \in D_{L} \mid u_{k}(x) \geq k\right\}\right| \leq k^{-q} \int_{D_{L}}\left|u_{k}\right|^{q} d x \leq C k^{-q / 2} .
$$

This is a weak- $L^{p}\left(D_{L}\right)$ estimate on $u^{+}=\max (u, 0)$, for $p=q / 2=d /(d-2)$ :

$$
\left\|u^{+}\right\|_{L_{W}^{p}\left(D_{L}\right)}=\sup _{t>0} t\left|\left\{x \in D_{L}|| u^{+}(x) \mid>t\right\}\right|^{1 / p} \leq C
$$

where the constant $C$ is independent of $L$ and $\beta \geq 0$.

Now let $\alpha \in(1, p), x_{0} \in D_{L}, R<\operatorname{dist}\left(x_{0}, y\right)$. The weak bound (5.92) implies that $u^{+} \in$ $L^{\alpha}\left(B_{R}\left(x_{0}\right)\right)$. By using the identity

$\int_{B_{R}}\left|u^{+}\right|^{\alpha} d x=\alpha \int_{0}^{\infty} t^{\alpha-1}\left|\left\{x \in B_{R} \mid u^{+}(x) \geq t\right\}\right| d t \leq\left|B_{R}\right| s^{\alpha}+\alpha \int_{s}^{\infty} t^{\alpha-1}\left|\left\{x \in B_{R} \mid u^{+}(x) \geq t\right\}\right| d t$ and optimizing in $s$, we see that

$$
\left\|u^{+}\right\|_{L^{\alpha}\left(B_{R}\right)} \leq C\left(\frac{p}{p-\alpha}\right)^{1 / \alpha}\left|B_{R}\right|^{\frac{p-\alpha}{p \alpha}}
$$

where the constant $C$ depends on $\alpha$ and $p$, but not on $L$ or $R$ or $\beta \geq 0$. Since $-\nabla \cdot(a \nabla u)+\beta u=$ $-\left|D_{L}\right|^{-1}$ in $B_{R}$, the estimates of De Giorgi and Moser give us a bound on $u^{+}(x)$ in terms of $\left\|u^{+}\right\|_{L^{\alpha}\left(B_{R}\left(x_{0}\right)\right)}$. Specifically, Theorem 4.1 of [21] (or Theorem 8.17 of [13]) implies that $u$ is locally bounded and satisfies:

$$
\sup _{x \in B_{R / 2}\left(x_{0}\right)} u^{+}(x) \leq C R^{-d / \alpha}\left(\int_{B_{R}}\left(u^{+}(y)\right)^{\alpha} d y\right)^{1 / \alpha}+C R^{2}\left|D_{L}\right|^{-1},
$$

with a constant $C$ that depends only on $d, a_{*}, a^{*}$, and $\alpha$. Note that in Theorem 4.1 of [21], the constant depends on $|\beta| R^{2}$. However, it is easy to see from the proof (method 1 ) that if $\beta$ is known to be non-negative, then the bound is independent of $\beta$, so the same bound holds under rescaling (as in Theorem 4.14 of [21]).

By combining (5.93) and (5.94) we have

$$
\sup _{x \in B_{R / 2}\left(x_{0}\right)} u^{+}(x) \leq C R^{-d / \alpha}\left|B_{R}\right|^{\frac{p-\alpha}{p \alpha}}+C R^{2}\left|D_{L}\right|^{-1} \leq C R^{-d / p}+C R^{2} L^{-d} \leq C R^{2-d},
$$


where the constant $C$ depends on the dimension, but not on $L, \beta \geq 0, R$. In particular,

$$
u^{+}(x) \leq C(\operatorname{dist}(x, y))^{2-d} .
$$

Now, assuming (5.87) holds for $u$ (otherwise, replace $u$ by $-u$ ), let us choose $r \leq 0$ such that both

$$
\left|\left\{x \in D_{L} \mid u(x)>r\right\}\right| \leq \frac{1}{2}\left|D_{L}\right|
$$

and

$$
\left|\left\{x \in D_{L} \mid u(x)<r\right\}\right| \leq \frac{1}{2}\left|D_{L}\right|
$$

hold. Consider the function $\bar{u}=r-u$ which satisfies

$$
-\nabla \cdot(a \nabla \bar{u})+\beta \bar{u}=-\delta_{y}+\left|D_{L}\right|^{-1}-\beta|r|
$$

and

$$
\left|\left\{x \in D_{L} \mid \bar{u}(x)>0\right\}\right| \leq \frac{1}{2}\left|D_{L}\right| .
$$

To the functions $\bar{u}_{k}=\max (0, \min (\bar{u}, k))$ and $\bar{u}^{+}=\max (0, \bar{u})$ we apply the same argument used to obtain (5.95). The result is:

$$
\bar{u}^{+}(x) \leq C(\operatorname{dist}(x, y))^{2-d} .
$$

In deriving (5.94) for $\bar{u}^{+}$, we must use the fact that $\bar{u}$ is a subsolution of $-\nabla \cdot(a \nabla \bar{u})+\beta \bar{u}=\left|D_{L}\right|^{-1}$ away from $y$, since $-\beta|r| \leq 0$. That is,

$$
\int_{B_{R}} \nabla \varphi \cdot a \nabla \bar{u}+\beta \bar{u} \varphi d x \leq\left|D_{L}\right|^{-1} \int_{B_{R}} \varphi d x
$$

holds for all $\varphi \in H_{0}^{1}\left(B_{R}\right)$ which satisfy $\varphi \geq 0$. Thus, Theorem 4.1 of [21] (or Theorem 8.17 of [13]) still applies. Apart from this detail, the argument is identical. By combining (5.95) and (5.96) we obtain

$$
r-C(\operatorname{dist}(x, y))^{2-d} \leq r-\bar{u}^{+}(x) \leq u(x) \leq u^{+}(x) \leq C(\operatorname{dist}(x, y))^{2-d}
$$

On the other hand, (5.95) implies that

$$
\int_{D_{L}} u^{+}(x) d x \leq C L^{2}
$$

We combine this with the fact that $\int_{D_{L}} u d x=0$ to conclude that

$$
\begin{aligned}
0 & =\int_{D_{L}} u^{+}(x) d x+\int_{\{r<u \leq 0\}} u(x) d x+\int_{\{u \leq r\}} u(x) d x \\
& \leq C L^{2}+r\left|\left\{x \in D_{L} \mid u(x) \leq r\right\}\right| \\
& =C L^{2}+r\left|\left\{x \in D_{L} \mid \bar{u}(x) \geq 0\right\}\right| \leq C L^{2}+r L^{d} / 2 .
\end{aligned}
$$

Hence $|r| \leq 2 C L^{2-d}$. Combining this with (5.97) we obtain $|u(x)| \leq C \operatorname{dist}(x, y)^{2-d}$, as desired. 


\section{$d=2:$ Proof of Lemma 4.5}

Lemma 4.5 relies on the following oscillation estimate, which is a version of Lemma 2.8(i) of [17]:

Lemma 5.1 Let $d=2$. For any $q \geq 1$, there is a constant $C>0$ such that

$$
R^{-2} \int_{B_{R}\left(x_{0}\right)}\left|G(x, y)-\bar{G}_{R}(y)\right|^{q} d x \leq C
$$

holds for all $x_{0} \in D_{L}, y \in D_{L} \backslash B_{2 R}\left(x_{0}\right), R>0, L>1$ and $\beta \geq 0$, where $\bar{G}_{R}(y)$ is the average of $G(\cdot, y)$ over the ball $B_{R}\left(x_{0}\right)$.

Proof of Lemma 5.1; This is proved as in Lemma 2.8 of [17] (see part (i), Step 2) for the freespace Green's function (see Step 2 in the proof therein); here we include the proof for completeness. Fix $y \in D_{L}$. Let $u(x)=G(x, y)$, which satisfies

$$
-\nabla \cdot(a \nabla u)+\beta u=\delta_{y}-\left|D_{L}\right|^{-1} .
$$

Let $\overline{u_{R}}$ be the average of $u$ over the ball $B_{R}$. Without loss of generality, suppose $\overline{u_{R}} \geq 0$. For $k \geq 0$, define

$$
u_{k}=\max \left(\min \left(u, \overline{u_{R}}+k\right), \overline{u_{R}}-k\right) .
$$

We claim that

$$
\int_{D_{L}}\left|\nabla u_{k}\right|^{2} d x \leq \frac{2 k}{a_{*}}
$$

To see this, observe that for any constant $c \in \mathbb{R}$,

$$
\begin{aligned}
\int_{D_{L}} \nabla u_{k} \cdot a \nabla u_{k} d x & =\int_{D_{L}} \nabla\left(u_{k}+c\right) \cdot a \nabla u d x \\
& =u_{k}(y)-\left|D_{L}\right|^{-1} \int_{D_{L}} u_{k}(x) d x-\beta \int_{D_{L}} u\left(u_{k}+c\right) d x
\end{aligned}
$$

If $\overline{u_{R}} \in[0, k]$, let $c=0$. Then $u(x)\left(u_{k}(x)+c\right) \geq 0$ at every point $x \in D_{L}$. Hence

$$
\beta \int_{D_{L}} u\left(u_{k}+c\right) d x \geq 0
$$

Therefore, (5.98) follows from (5.99). If $\overline{u_{R}}>k$, let $c=k-\overline{u_{R}}$. Then $u_{k}+c \geq 0$. Also, $u(x)>\overline{u_{R}}-$ $k>0$ must hold wherever $\left(u_{k}(x)+c\right)>0$. Hence (5.100) still holds. Moreover, $0 \leq u_{k}(x)+c \leq 2 k$, so again (5.98) follows from (5.99).

Now let $v(x)=u(x)-\overline{u_{R}}$. Let $v_{k}(x)=\max (\min (v(x), k),-k)=u_{k}(x)-\overline{u_{R}}$. Let $\overline{v_{R}}$ and $\overline{v_{k, R}}$ be the average of $v$ and $v_{k}$ over $B_{R}$, respectively. Hence $\overline{v_{R}}=0$. Then the goal is to bound

$$
\begin{aligned}
\left(R^{-2} \int_{B_{R}}|v|^{q} d x\right)^{1 / q}= & \left(R^{-2} \int_{B_{R} \cap\{|v| \leq k\}}\left|v_{k}\right|^{q} d x+R^{-2} \int_{B_{R} \cap\{|v|>k\}}|v|^{q} d x\right)^{1 / q} \\
\leq & C\left(R^{-2} \int_{B_{R} \cap\{|v| \leq k\}}\left|v_{k}-\overline{v_{k, R}}\right|^{q} d x\right)^{1 / q}+C\left|\overline{v_{k, R}}\right| \\
& +C\left(R^{-2} \int_{B_{R} \cap\{|v|>k\}}|v|^{q} d x\right)^{1 / q} .
\end{aligned}
$$


Since $\overline{v_{R}}=0$, we have

$$
\left|\overline{v_{k, R}}\right| \leq 2\left(R^{-2} \int_{B_{R} \cap\{|v| \geq k\}}|v|^{q} d x\right)^{1 / q}
$$

Therefore,

$$
\begin{aligned}
\left(R^{-2} \int_{B_{R}}|v|^{q} d x\right)^{1 / q} \leq & C\left(R^{-2} \int_{B_{R}}\left|v_{k}-\overline{v_{k, R}}\right|^{q} d x\right)^{1 / q} \\
& +C\left(R^{-2} \int_{B_{R} \cap\{|v|>k\}}|v|^{q} d x\right)^{1 / q} .
\end{aligned}
$$

By the Sobolev inequality and then (5.98), we know that for any $s \in[1, \infty)$ there is a constant $C_{s}$ (depending only on $s$ ) such that

$$
\left(R^{-2} \int_{B_{R}}\left|v_{k}-\overline{v_{k, R}}\right|^{s} d x\right)^{1 / s} \leq C_{s}\left(\int_{B_{R}}\left|\nabla v_{k}\right|^{2} d x\right)^{1 / 2}=C_{s}\left(\int_{B_{R}}\left|\nabla u_{k}\right|^{2} d x\right)^{1 / 2} \leq C k^{1 / 2} .
$$

To estimate the last integral appearing in (5.101) we use

$$
\int_{B_{R} \cap\{|v|>k\}}|v|^{q} d x=\int_{0}^{\infty} q t^{q-1}|\{|v| \geq \max (t, k)\}| d t \leq|\{|v| \geq k\}| k^{q}+q \int_{k}^{\infty} t^{q-1}|\{|v| \geq t\}| d t
$$

and

$$
|\{|v| \geq k\}| \leq\left|\left\{\left|v_{k}\right| \geq k\right\}\right| \leq k^{-s} \int_{B_{R}}\left|v_{k}\right|^{s} d x
$$

Let $s>2 q$. Then

$$
\begin{aligned}
\int_{B_{R}}\left|v_{k}\right|^{s} d x & \leq C \int_{B_{R}}\left|v_{k}-\overline{v_{k, R}}\right|^{s} d x+C R^{2}\left(\overline{v_{k, R}}\right)^{s} \\
& \leq C \int_{B_{R}}\left|v_{k}-\overline{v_{k, R}}\right|^{s} d x+C R^{2}\left(R^{-2} \int_{B_{R}}|v|^{q} d x\right)^{s / q} \\
& \leq C R^{2} k^{s / 2}+C R^{2}\left(R^{-2} \int_{B_{R}}|v|^{q} d x\right)^{s / q} .
\end{aligned}
$$

So, if $I_{q}=\left(R^{-2} \int_{B_{R}}|v|^{q} d x\right)^{1 / q}$, we have

$$
\int_{B_{R}}\left|v_{k}\right|^{s} d x \leq C R^{2} k^{s / 2}+C R^{2} I_{q}^{s}
$$

and $|\{|v| \geq k\}| \leq R^{2} k^{-s / 2}+C k^{-s} R^{2} I_{q}^{s}$. 
Combining these bounds and returning to (5.101), we obtain

$$
\begin{aligned}
I_{q} \leq & C k^{1 / 2}+C R^{-2 / q}\left(|\{|v| \geq k\}| k^{q}+q \int_{k}^{\infty} t^{q-1}|\{|v| \geq t\}| d t\right)^{1 / q} \\
\leq & C k^{1 / 2}+C R^{-2 / q}\left(R^{2} k^{-s / 2} k^{q}+C k^{-s} R^{2} I_{q}^{s} k^{q}\right)^{1 / q} \\
& +C R^{-2 / q}\left(q \int_{k}^{\infty} t^{q-1}|\{|v| \geq t\}| d t\right)^{1 / q} \\
\leq & C k^{1 / 2}+C R^{-2 / q}\left(R^{2} k^{-s / 2} k^{q}+C k^{-s} R^{2} I_{q}^{s} k^{q}\right)^{1 / q} \\
& +C R^{-2 / q}\left(q \int_{k}^{\infty} t^{q-1}\left(R^{2} t^{-s / 2}+C t^{-s} R^{2} I_{q}^{s}\right) d t\right)^{1 / q} \\
\leq & C k^{1 / 2}+C k^{1-s /(2 q)}+C I_{q}^{s / q} k^{1-s / q} .
\end{aligned}
$$

By choosing $k=\alpha I_{q}$ with $\alpha>0$ sufficiently large, we see that this implies $I_{q} \leq C$.

Now we continue with the proof of Lemma 4.5. By assumption, $\operatorname{dist}\left(x_{0}, y\right)>2 R$. Let $\varphi$ be a smooth function supported in $B_{2 R}\left(x_{0}\right)$ and satisfying: $0 \leq \varphi(x) \leq 1$ for all $x, \varphi(x)=1$ for $x \in B_{R}\left(x_{0}\right)$, and $|\nabla \varphi| \leq C / R$. Applying Lemma 3.2 to $u(x)=G(x, y)$ with this choice of $\varphi$, we conclude

$$
\begin{aligned}
\int_{B_{R}}|\nabla u|^{2} d x \leq & K_{1}\left|D_{L}\right|^{-1} \int_{B_{2 R}}(u-b) \varphi^{2} d x-K_{1} \beta \int_{B_{2 R}} u(u-b) \varphi^{2} d x \\
& +K_{2} \int_{B_{2 R}}|\nabla \varphi|^{2}(u-b)^{2} d x
\end{aligned}
$$

If we choose

$$
b=\left(\int_{B_{2 R}} \varphi^{2} d x\right)^{-1} \int_{B_{2 R}} u \varphi^{2} d x
$$

then Jensen's inequality implies

$$
\int_{B_{2 R}} u(u-b) \varphi^{2} d x \geq 0
$$

Therefore, since $\beta \geq 0$,

$$
\begin{aligned}
\int_{B_{R}}|\nabla u|^{2} d x \leq & K_{1}\left|D_{L}\right|^{-1} \int_{B_{2 R}}(u-b) \varphi^{2} d x+K_{2} \int_{B_{2 R}}|\nabla \varphi|^{2}(u-b)^{2} d x \\
& \leq K_{1} R^{-2} \int_{B_{2 R}}|u-b| d x+C R^{-2} \int_{B_{2 R}}(u-b)^{2} d x .
\end{aligned}
$$

On the other hand, if $\bar{u}$ denotes the average of $u(x)$ over $B_{2 R}\left(x_{0}\right)$, we know from Lemma 5.1 that

$$
R^{-2} \int_{B_{2 R}}|u-\bar{u}| d x \leq C .
$$

Hence

$$
|\bar{u}-b| \leq\left(\int_{B_{2 R}} \varphi^{2} d x\right)^{-1} \int_{B_{2 R}}|\bar{u}-u(x)| \varphi^{2} d x \leq C R^{-2} \int_{B_{2 R}}|\bar{u}-u(x)| \varphi^{2} d x \leq C .
$$


Applying Lemma 5.1 again, we obtain

$$
R^{-2} \int_{B_{2 R}}(u-b)^{2} d x \leq C R^{-2} \int_{B_{2 R}}(u-\bar{u})^{2} d x+C R^{-2} \int_{B_{2 R}}(\bar{u}-b)^{2} d x \leq C .
$$

Similarly,

$$
R^{-2} \int_{B_{2 R}}|u-b| d x \leq C R^{-2} \int_{B_{2 R}}|u-\bar{u}| d x+C R^{-2} \int_{B_{2 R}}(\bar{u}-b) d x \leq C .
$$

In view of (5.104) and the fact that $C$ is independent of $R, L$ and $\beta \geq 0$, we have proved the desired result.

\section{References}

[1] G. Bal, J. Garnier, S. Motsch, V. Perrier, Random integrals and correctors in homogenization Asympt. Anal. 59 (2008), pp. 1-26.

[2] S. M. Bergin, A. R. Rathmell, Y.-H. Chen, P. Charbonneau, Z.-Y. Li and B. J. Wiley, The effect of nanowire length and diameter on the properties of transparent, conducting nanowire films, Nanoscale 4 (2012), pp. 1996-2004.

[3] M. Biskup, Recent progress on the random conductance model, Prob. Surveys 8 (2011), pp. 294-373.

[4] M. Biskup, M. Salvi, and T. Wolff, A central limit theorem for the effective conductance: Linear boundary data and small ellipticity contrasts, Comm. Math. Phys. 328 (2014), pp. 701-731.

[5] D. Boivin, Tail estimates for homogenization theorems in random media, ESAIM Probab. Stat. 13 (2009), pp. 51-69.

[6] A. Bourgeat and A. Piatnitski, Approximations of effective coefficients in stochastic homogenization, Ann. I.H. Poincaré 40 (2004), pp. 153-165.

[7] A. Bourgeat and A. Piatnitski, Estimates in probability of the residual between the random and the homogenized soutions of one-dimensional second-order operator, Asympt. Anal. 21 (1999), pp. 303-315.

[8] S. Chatterjee, A new method of normal approximation, Ann. Prob. 36 (2008), pp. 1584-1610.

[9] S. Chatterjee, Fluctuations of eigenvalues and second order Poincaré inequalities, Prob. Theory Relat. Fields. 143 (2009), pp. 1-40.

[10] J. Conlon and A. Naddaf, Green's functions for elliptic and parabolic equations with random coefficients, New York J. Math. 6 (2000), pp. 153-225.

[11] T. Delmotte and J.-D. Deuschel On estimating the derivatives of symmetric diffusions in stationary random environments, with applications to the $\nabla \phi$ interface model. Probab. Theory Relat. Fields, 133 (2005), pp. 358-390.

[12] B. Efron and C. Stein, The jackknife estimate of variance, Annals of Statistics 9 (1981) pp. 586-596. 
[13] D. Gilbarg and N. Trudinger, Elliptic Partial Differential Equations of Second Order, Springer, 2001.

[14] M. Giaquinta, Multiple Integrals in the Calculus of Variations and Nonlinear Elliptic Systems, Princeton University Press, 1983.

[15] A. Gloria, S. Neukamm, F. Otto. Quantification of ergodicity in stochastic homogenization: optimal bounds via spectral gap on Glauber dynamics, Inventiones Mathematicae, 2014. http://dx.doi.org/10.1007/s00222-014-0518-z

[16] A. Gloria, Fluctuation of Solutions to Linear Elliptic Equations with Noisy Diffusion Coefficients, Comm. PDE, 38 (2012), pp. 304-338.

[17] A. Gloria and F. Otto, An optimal variance estimate in stochastic homogenization of discrete elliptic equations, Ann. Probability 39 (2011), pp. 779-856.

[18] A. Gloria and F. Otto, An optimal error estimate in stochastic homogenization of discrete elliptic equations, Ann. Appl. Probability 22 (2012), pp. 1-28.

[19] A. Gloria and F. Otto, Quantitative results on the corrector equation in stochastic homogenization, in preparation, 2014.

[20] M. Grüter and Kjell-Ove Widman, The Green function for uniformly elliptic equations, Manuscripta Math. 37 (1982), pp. 303-342.

[21] Q. Han and F. Lin, Elliptic Partial Differential Equations. 2nd Ed. Courant Lecture Notes, American Mathematical Society, 2011.

[22] V.V. Jikov, S.M. Kozlov, O.A. Oleinik, Homogenization of differential operators and integral functionals. Springer-Verlag, New York: 1994.

[23] T. Komorowski and L. Ryzhik, A sharp bound on the $L^{2}$ norm of the solution of a random elliptic difference equation. Commun. Math. Sci. 9 (2011), pp. 607-622.

[24] S.M. Kozlov, The averaging of random operators, Math. USSR Sb. 109 (1979), pp. 188-202.

[25] D. Marahrens and F. Otto, Annealed estimates on the Green function, Preprint, 2013. http://arxiv.org/abs/1304.4408

[26] N. Meyers, An $L^{p}$-estimate for the gradient of solutions of second order elliptic divergence equations. Ann. Scuola Norm. Sup. Pisa, Classe di Scienze, 17 (1963), pp. 189-206.

[27] J.-C. Mourrat and F. Otto, Correlation structure of the correcto in stochastic homogenization, preprint 2014. http://arxiv.org/abs/1402.1924

[28] A. Naddaf and T. Spencer, Estimates on the variance of some homogenization problems, unpublished manuscript, 1998.

[29] J. Nolen, Normal approximation for a random elliptic equation, Probab. Theory Relat. Fields, 2013. http://dx.doi.org/10.1007/s00440-013-0517-9

[30] J. Nolen and F. Otto, In preparation, 2014.

[31] H. Owhadi, Approximation of the effective conductivity of ergodic media by periodization, Probab. Theory Relat. Fields 125 (2003), pp. 225-258. 
[32] G.C. Papanicolaou and S.R.S. Varadhan, Boundary value problems with rapidly oscillating random coefficients. in Random Fields Vol. I, II (Esztergom, 1979), Colloq. Math. Soc. János Bolyai, 27 North Holland, New York: 1981. pp. 835- 873.

[33] R. Rossignol, Noise-stability and central limit theorems for effective resistance of random electric networks, preprint (2012), http://arxiv.org/abs/1206.3856

[34] J.M. Steele, An Efron-Stein inequality for nonsymmetric statistics, Ann. Statistics, 14 (1986), pp. 753-758.

[35] C. Stein, Approximate Computation of Expectations. IMS Lecture Notes - Monograph Series 7. IMS, Hayward, CA., 1972.

[36] S. Torquato, Random Heterogeneous Materials, Springer, 2001.

[37] J. Wehr, A lower bound on the variance of conductance in random resistor networks, J. Stat. Phys, 86 (1997), No. 5,6, pp. 1359-1365.

[38] V.V. Yurinskii, Averaging of symmetric diffusion in a random medium, Siberian Math. J. 4 (1986), pp. 603-613. 